\title{
ON LIKELY SOLUTIONS OF THE STABLE MATCHING PROBLEM WITH UNEQUAL NUMBERS OF MEN AND WOMEN
}

\author{
BORIS PITTEL
}

\begin{abstract}
Following up a recent work by Ashlagi, Kanoria and Leshno, we study a stable matching problem with unequal numbers of men and women, and independent uniform preferences. The asymptotic formulas for the expected number of stable matchings, and for the probabilities of one point-concentration for the range of husbands' total ranks and for the range of wives' total ranks are obtained.
\end{abstract}

\section{INTRODUCTION AND MAIN RESULTS}

Consider the set of $n_{1}$ men and $n_{2}$ women facing a problem of selecting a marriage partner. For $n_{1}=n_{2}=n$, a marriage $\mathcal{M}$ is a matching (bijection) between the two sets. It is assumed that each man and each woman has his/her preferences for a marriage partner, with no ties allowed. That is, there are given $n$ permutations $\sigma_{j}$ of the men set and $n$ permutations $\rho_{j}$ of the women set, each $\sigma_{j}\left(\rho_{j}\right.$ resp.) ordering the women set (the men set resp.) according to the desirability degree of a woman (a man) as a marriage partner for man $j$ (woman $j$ ). A marriage is called stable if there is no unmarried pair (a man, a woman) who prefer each other to their respective partners in the marriage. A classic theorem, due to Gale and Shapley [4, asserts that, given any system of preferences $\left\{\rho_{j}, \sigma_{j}\right\}_{j \in[n]}$, there exists at least one stable marriage $\mathcal{M}$.

The proof of this theorem is algorithmic. A bijection is constructed in steps such that at each step every man not currently on hold makes a proposal to his best choice among women who haven't rejected him before, and the chosen woman either provisionally puts the man on hold or rejects him, based on comparison of him to her current suitor if she has one already. Since a woman who once gets proposed to always has a man on hold afterwards, after finally many steps every woman has a suitor, and the resulting bijection turns out to be stable. Of course the roles can be reversed, with

Date: February 14, 2017.

2010 Mathematics Subject Classification. 05C30, 05C80, 05C05, 34E05, 60C05.

Key words and phrases. stable matching, random preferences, asymptotics . 
women proposing and each man selecting between the current proponent and a woman whose proposal he currently holds, if there is such a woman. In general, the two resulting matchings, $\mathcal{M}_{1}$ and $\mathcal{M}_{2}$ are different, one manoptimal, another woman-optimal. "Man/woman-optimal" means that each man/woman is matched with the best woman/ man among all his/her stable women/men, i.e. those who are the man's/woman's partner in at least one stable matching. Strikingly, the man-optimal (woman-optimal) stable matching is woman-pessimal (man-pessimal), meaning that every woman (man) is matched to her/his worst stable husband (wife). The interested reader is encouraged to consult Gusfield and Irving [5] for a rich, detailed analysis of the algebraic (lattice) structure of stable matchings set, and a collection of proposal algorithms for determination of stable matchings in between the two extremal matchings $\mathcal{M}_{1}$ and $\mathcal{M}_{2}$.

A decade after the Gale-Shapley paper, McVitie and Wilson [12] developed an alternative, sequential, algorithm in which proposals by one side to another are made one at a time. This procedure delivers the same matching as the Gale-Shapley algorithm; the overall number of proposals made, say by men to women, is clearly the total rank of the women in the terminal matching.

This purely combinatorial, numbers-free, description begs for a probabilistic analysis of the problem chosen uniformly at random among all the instances, whose total number is $(n !)^{2 n}$. Equivalently the $2 n$ preference permutations $s_{j}$ and $\sigma_{j}$ are uniform, and independent. In a pioneering paper [16] Wilson reduced the work of the sequential algorithm to a classic urn scheme (coupon-collector problem) and proved that the expected running time, whence the expected total rank of wives in the man-optimal matching, is at most $n H_{n} \sim n \log n, H_{n}=\sum_{j=1}^{n} 1 / j$.

Few years later Knuth [9], among other results, found a better upper bound $(n-1) H_{n}+1$, and established a matching lower bound $n H_{n}-$ $O\left(\log ^{4} n\right)$. He also posed a series of open problems, one of them on the expected number of the stable matchings. Knuth pointed out that an answer might be found via his formula for the probability $P(N)$ that a generic matching $\mathcal{M}$ is stable:

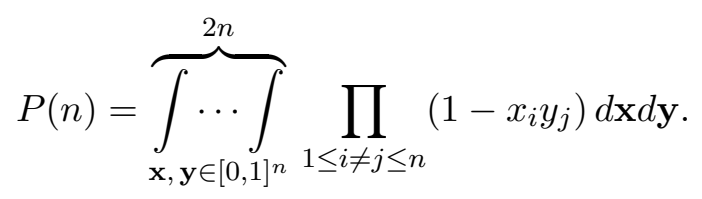

(His proof relied on an inclusion-exclusion formula, and interpretation of each summand as the value of a $2 n$-dimensional integral, with the integrand equal to the corresponding summand in the expansion of the integrand in 
(1.1).) And then the expected value of $S(n)$, the total number of stable matchings, would then be determined from $\mathrm{E}[S(n)]=n ! P(n)$.

Following Don Knuth's suggestion, in [13] we used the equation (1.1) to obtain an asymptotic formula

$$
P(n)=(1+o(1)) \frac{e^{-1} n \log n}{n !},
$$

which implied that $\mathrm{E}[S(n)] \sim e^{-1} n \log n$. We also found the integral formulas for $P_{k}(n)\left(P_{\ell}(n)\right.$ resp.) the probability that the generic matching $\mathcal{M}$ is stable and that the total man-rank $R(\mathcal{M})$ (the total woman-rank $Q(\mathcal{M})$ is $\ell$ resp.). These integral formulas implied that with high probability (w.h.p. from now) for each stable matching $\mathcal{M}$ the ranks $R(\mathcal{M}), Q(\mathcal{M})$ are between $(1+o(1)) n \log n$ and $(1+o(1)) n^{2} / \log n$. It followed, with some work, that w.h.p. $R\left(\mathcal{M}_{1}\right) \sim n^{2} / \log n, Q\left(\mathcal{M}_{1}\right) \sim n \log n$ and $R\left(\mathcal{M}_{2}\right) \sim n \log n$, $Q\left(\mathcal{M}_{2}\right) \sim n^{2} / \log n$. In particular, w.h.p. $R\left(\mathcal{M}_{j}\right) Q\left(\mathcal{M}_{j}\right) \sim n^{3},(j=1,2)$. In a joint paper with Knuth and Motwani [10] we used a novel extension of Wilson's proposal algorithm to show that every woman w.h.p. has at least $(1 / 2-o(1)) \log n$ stable husbands.

Spurred by these results, in [14] we studied the likely behavior of the full random set $\{(R(\mathcal{M}), Q(\mathcal{M}))\}$, where $\mathcal{M}$ runs through all stable matchings for the random instance of $\left\{\rho_{j}, \sigma_{j}\right\}_{j \in[n]}$. The key ingredient was the more general formula for $P_{k, \ell}(n)$, the probability that the generic matching $\mathcal{M}$ is stable and $Q(\mathcal{M})=k, R(\mathcal{M})=\ell$. We also showed that, for a generic woman, the number of stable husbands is normal in the limit, with mean and variance asymptotic to $\log n$.

The key element of the proofs of the integral representations for these probabilities, which also imply the Knuth formula (1.1), was a refined, background, probability space. Its sample point is a pair of two $n \times n$ matrices $\mathbf{X}=\left\{X_{i, j}\right\}, \mathbf{Y}=\left\{Y_{i, j}\right\}$ with all $2 n^{2}$ entries being independent, [0,1]-uniform random variables. Reading each row of $\mathbf{X}$ and each column of $\mathbf{Y}$ in increasing order we recover the independent, uniform preferences of each of $n$ men and of each of $n$ women respectively. And, for instance, the integrand in (1.1) turns out equal to the probability that a generic matching $M$ is stable, conditioned on the values $x_{i}=X_{i, M(i)}, y_{j}=Y_{M^{-1}(j), j}$

Using the formula for $P_{k, \ell}(n)$, we proved a law of hyperbola: for every $\lambda \in\left(0,1 / 4\right.$ ), quite surely (q.s) $\max _{\mathcal{M}}\left|n^{-3} Q(\mathcal{M}) R(\mathcal{M})-1\right| \leq n^{-\lambda}$; "quite surely" means with probability $1-O\left(n^{-K}\right)$, for every $K$, a notion introduced in Knuth, Motwani and Pittel [10].

Moreover, q.s. every point on the hyperbolic arc $\{(u, v): u v=1 ; u, v \in$ $\left.\left[n^{-\lambda}, n^{\lambda}\right]\right\}$ is within distance $n^{-(1 / 4-\lambda)}$ from $n^{-3 / 2}(Q(\mathcal{M}), R(\mathcal{M}))$ for some stable matching $\mathcal{M}$. In particular, q.s. $S(n) \geq n^{1 / 2-o(1)}$, a significant 
improvement of the logarithmic bound in [10], but still far below $n \log n$, the asymptotic order of $\mathrm{E}\left[S_{n}\right]$.

Thus, for a large number of participants, a typical instance of the preferences $\left\{\rho_{j}, \sigma_{j}\right\}_{j \in[n]}$ has multiple stable matchings very nearly obeying the preservation law for the product of the total man-rank and the total womanrank. In a way this law is not unlike thermodynamic laws in physics of gases. However those laws are usually of phenomenological nature, while the product law is a rigorous corollary of the local stability conditions for the random instance of the preferences $\left\{\rho_{j}, \sigma_{j}\right\}_{j \in[n]}$.

The hyperbola law implied that w.h.p. the minimum value of $R(\mathcal{M})+$ $Q(\mathcal{M})$ (by definition attained at an egalitarian marriage $\mathcal{M}_{3}$ ) is asymptotic to $2 n^{3 / 2}$, and the worst spouse rank in $\mathcal{M}_{3}$ is of order $n^{1 / 2} \log n$, while the worst spouse rank in the extremal $\mathcal{M}_{1}$ and $\mathcal{M}_{2}$ is much larger, of order $n / \log n$.

Recently Lennon and Pittel [11] extended the techniques in [13, [14] to show that $\mathrm{E}\left[S(n)^{2}\right] \sim\left(e^{-2}+0.5 e^{-3}\right)(n \log n)^{2}$. Combined with (1.2), this result implied that $S(n)$ is of order $n \log n$ with probability 0.84 , at least. Jointly with Shepp and Veklerov [15] we proved that, for a fixed $k$, the expected number of women with $k$ stable husbands is asymptotic to $(\log n)^{k+1} /(k-1)$ !.

We hope the reader shares our view that the case of the uniform preferences turned out to be surprisingly amenable to the asymptotic analysis, and as such it can serve a benchmark for more general models that might be closer to "real-life" situations.

In this paper we will consider a matching model with sets of men and women of different cardinalities $n_{1}$ and $n_{2}$, say $n_{1}<n_{2}$. In this case Gusfield and Iriving [5] defined a stable matching as an injection $\mathcal{M}:\left[n_{1}\right] \rightarrow\left[n_{2}\right]$ such that there is no unmatched pair $(m, w),\left(m \in\left[n_{1}\right], w \in\left[n_{2}\right]\right)$, meeting a condition:

$m$ prefers $w$ to his partner in $\mathcal{M}$, and if $w$ is matched in $\mathcal{M}$ then $w$ prefers $m$ to her partner in $\mathcal{M}$.

It was demonstrated in [5] that, for any preference lists, at least one stable matching (injection) exists, and the women set $\left[n_{2}\right]$ is partitioned into two subsets $A_{1}$ and $A_{2},\left(\left|A_{1}\right|=n_{1},\left|A_{2}\right|=n_{2}-n_{1}\right)$ such that the women from $A_{1}$ are matched in all stable matchings, and the women from $A_{2}$ in none.

Few years ago Ashlagi, Kanoria and Leshno [1] (see Online Appendices $\mathrm{A}, \mathrm{B}$ and $\mathrm{C}$ for the proofs) discovered that the mere positivity of $n_{2}-n_{1}$ drastically changes the likely structure of the stable matchings. Let $R(\mathcal{M})$ and $Q(\mathcal{M})$ continue to stand for the total rank of husbands and the total rank of wives in a stable matching $\mathcal{M}$. Their main result states: 
Theorem 1.1. (Ashlagi, Kanoria, Leshno, (AKL)) Let $n_{1}, n_{2} \rightarrow \infty$ and $n_{2}-n_{1}>0$. For every $\varepsilon>0$, w.h.p. (1) for every two stable matchings $M$ and $M^{\prime}$ both $R(M) / R\left(M^{\prime}\right)$ and $Q(M) / Q\left(M^{\prime}\right)$ are $1+O(\varepsilon)$, uniformly over $M$ and $M^{\prime}$; (2) denoting $s(\mathbf{n})=\log \frac{n_{2}}{n_{2}-n_{1}}$,

$$
\max _{\mathcal{M}} Q(\mathcal{M}) \leq(1+\varepsilon) n_{2} s(\mathbf{n}), \quad \min _{\mathcal{M}} R(M) \geq \frac{n_{1}^{2}}{1+(1+\varepsilon) \frac{n_{2}}{n_{1}} s(\mathbf{n})} .
$$

(3) the fraction of men and the fraction of women who have multiple stable partners are each no more than $\varepsilon$.

The contrast with the case $n_{1}=n_{2}=n$ is stark indeed. There w.h.p. a generic member has $\left(1+o_{p}(1)\right) \log n$ stable partners (see [10], [14]), and the ratios $R(\mathcal{M}) / R\left(\mathcal{M}^{\prime}\right), Q(\mathcal{M}) / Q\left(\mathcal{M}^{\prime}\right)$ reach the values asymptotic to $n /(\log n)^{2}$ and $(\log n)^{2} / n$, 14]. As stressed in [1], Theorem 1.1 implies that, as long as the focus is on the global parameters $R(\mathcal{M})$ and $Q(\mathcal{M})$ ("centralized markets"), the likely dependence on which side proposes almost vanishes. In the outline, I learned of Theorem 1.1 first from Jennifer Chayes [2], and later, with more details, from Gil Kalai's blog [7].

As a promising sign, the basic integral identities for the probabilities $P(n)$ (Knuth [9]), $P_{k}(n)$ and $P_{\ell}(n)$ (13], 14]) for the " $n_{1}=n_{2}=n$ " have natural counterparts for the "unbalanced" probabilities $P(\mathbf{n}), P_{k}(\mathbf{n})$ and $P_{\ell}(\mathbf{n}), \mathbf{n}:=\left(n_{1}, n_{2}\right)$. Here $P(\mathbf{n})$ is the probability that a generic injection $\mathcal{M}:\left[n_{1}\right] \rightarrow\left[n_{2}\right]$ is stable; $(2) P_{k}(\mathbf{n})\left(P_{\ell}(\mathbf{n})\right.$ resp. $)$ is the probability that a generic injection $\mathcal{M}:\left[n_{1}\right] \rightarrow\left[n_{2}\right]$ is stable and $Q(\mathcal{M})=k,(R(\mathcal{M})=\ell$ resp.). All three formulas are implied by the integral formula for $P_{k, \ell}(\mathbf{n})$, the probability that $\mathcal{M}$ is stable and $Q(\mathcal{M})=k, R(\mathcal{M})=\ell$. 


\section{Lemma 1.2.}

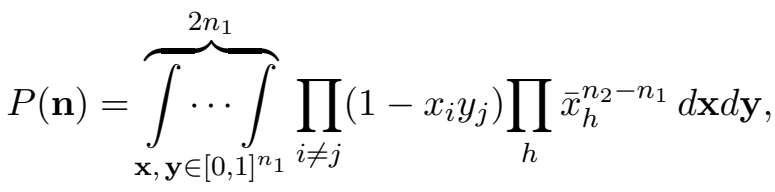

$$
\begin{aligned}
& P_{k}(\mathbf{n})=\overbrace{\int_{\mathbf{x}, \mathbf{y} \in[0,1]^{n_{1}}} \ldots \int^{2 n_{1}}\left[\xi^{k-n_{1}}\right]} \prod_{i \neq j}\left(1-x_{i}\left(1-\xi+\xi y_{j}\right)\right) \prod_{h} \bar{x}_{h}^{n_{2}-n_{1}} d \mathbf{x} d \mathbf{y},
\end{aligned}
$$

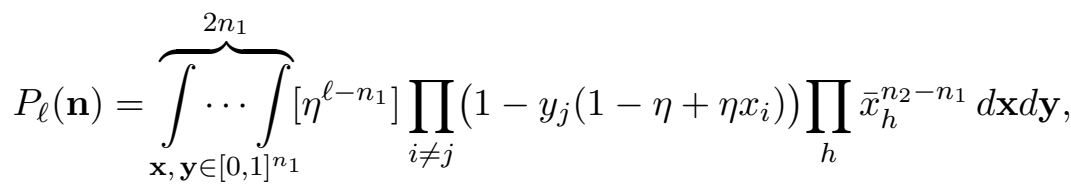

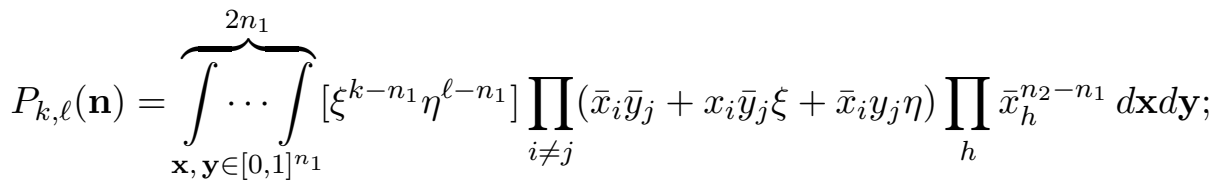

here $i, j, h \in\left[n_{1}\right]$ and $\bar{x}_{i}=1-x_{i}, \bar{y}_{j}=1-y_{j}$.

Thus the condition $n_{1}<n_{2}$ leads to insertion of the extra factor $\prod_{h} \bar{x}_{h}^{n_{2}-n_{1}}$ into the corresponding integrands for $P(n), P_{k}(n), P_{\ell}(n)$, and $P_{k, \ell}(n)$ for the the " $n_{1}=n_{2}=n$ " case in [14].

Using the formula for $P(\mathbf{n})$, and the fact that $\mathrm{E}[S(\mathbf{n})]=\left(\begin{array}{c}n_{2} \\ n_{1}\end{array}\right) n_{1} ! P(\mathbf{n})$, we will prove

Theorem 1.3. If $n_{2}>n_{1} \rightarrow \infty$ then

$$
\mathrm{E}[S(\mathbf{n})] \sim \frac{n_{1} \exp \left(-\frac{e^{s(\mathbf{n})}-1-s(\mathbf{n})}{e^{s(\mathbf{n})}-1}\right)}{\left(n_{2}-n_{1}\right) s(\mathbf{n})}, \quad s(\mathbf{n}):=\log \frac{n_{2}}{n_{2}-n_{1}} .
$$

Consequently, if $n_{2} \gg n_{2}-n_{1}>0$, i.e. $n_{2} / n_{1} \rightarrow 1$ and $s(\mathbf{n}) \rightarrow \infty$, then

$$
\mathrm{E}[S(\mathbf{n})] \sim e^{-1} \frac{n_{1}}{\left(n_{2}-n_{1}\right) \log n_{1}},
$$

and if $\frac{n_{2}}{n_{1}} \rightarrow \infty$, then $\mathrm{E}[S(\mathbf{n})] \rightarrow 1$. Finally if $\lim \frac{n_{2}}{n_{1}}=c>1$ is finite, then

$$
\lim \mathrm{E}[S(\mathbf{n})]=e^{-1} \frac{\lambda(c)}{\log \lambda(c)}, \quad \lambda(c)=\left(\frac{c}{c-1}\right)^{c-1} .
$$

Note. Recall that for $n_{2}=n_{1}$ we had proved that $\mathrm{E}[S(\mathbf{n})] \sim e^{-1} n_{1} \log n_{1}$. Thus increasing the cardinality of one of the sides just by 1 reduces the asymptotic expected number of stable matchings by the factor of $\log ^{2} n_{1}$, but the resulting number is a sizable $e^{-1} \frac{n_{1}}{\log n_{1}}$. 
We conjecture that for $n_{2} / n_{1} \rightarrow 1$, i.e. when $\mathrm{E}[S(\mathbf{n})] \rightarrow \infty$, the second order moment $\mathrm{E}\left[S^{2}(\mathbf{n})\right]=O\left(\mathrm{E}^{2}[S(\mathbf{n})]\right)$, so that, with a positive limiting probability, $S(\mathbf{n})=\theta(\mathrm{E}[S(\mathbf{n})])$, in a complete analogy with the case $n_{1}=$ $n_{2}$, see [11].

On the other hand, once the difference $n_{2}-n_{1}$ becomes comparable to the smaller cardinality, the limiting expected number is finite-still above 1 as it should be-implying that w.h.p. there are "just a few" stable matchings. Finally, if $n_{2} / n_{1} \rightarrow \infty$ then $\lim \mathrm{E}[S(\mathbf{n})]=1$, implying that $\lim \mathrm{P}(S(\mathbf{n})=$ 1) $=1$.

Our next two theorems establish sharp concentration of the likely ranges $\{Q(\mathcal{M})\}_{\mathcal{M}}$ and $\{R(\mathcal{M})\}_{\mathcal{M}}$ of husbands' and wives' ranks around certain deterministic functions of $\mathbf{n}$, when $\mathcal{M}$ runs through the set of all stable matchings. Let $a, b, c, d<1 / 2$, and

$$
\begin{aligned}
& \delta(\mathbf{n}):= \begin{array}{ll}
s(\mathbf{n})^{-b}, & \text { if } s(\mathbf{n}) \rightarrow \infty, \\
n_{1}^{-a}, & \text { if } s(\mathbf{n})=O(1),
\end{array} \\
& \mathcal{P}(\mathbf{n}):= \begin{cases}\exp \left(-c\left(n_{2}-n_{1}\right) s(\mathbf{n})^{2(1-b)}\right), & \text { if } s(\mathbf{n}) \rightarrow \infty, \\
\exp \left(-\theta\left(n_{1}^{1-2 a}\right)\right), & \text { if } s(\mathbf{n})=O(1) .\end{cases}
\end{aligned}
$$

Theorem 1.4. For $n_{2}>n_{1}$ and $n_{1}$ sufficiently large,

$$
\mathrm{P}\left(\max _{\mathcal{M}}\left|\frac{Q(\mathcal{M})}{n_{2} s(\mathbf{n})}-1\right| \geq \delta(\mathbf{n})\right) \leq \mathcal{P}(\mathbf{n}) .
$$

Next, introduce

$$
f(x)=\frac{e^{x}-1-x}{x\left(e^{x}-1\right)}
$$

and define

$$
\delta^{*}(\mathbf{n}):=\frac{\delta(\mathbf{n})}{s(\mathbf{n}) f(s(\mathbf{n}))}
$$

By (1.3), and $s(\mathbf{n}) \geq \frac{n_{1}}{n_{2}}$,

$$
\delta^{*}(\mathbf{n})= \begin{cases}O\left(s(\mathbf{n})^{-b}\right), & \text { if } s(\mathbf{n}) \rightarrow \infty, \\ O\left(n_{2} n_{1}^{-1-a}\right), & \text { if } s(\mathbf{n})=O(1),\end{cases}
$$

where $a, b<1 / 2$. The case $s(\mathbf{n})=O(1)$ forces us to impose the condition $n_{2} \leq n_{1}^{3 / 2-d}, d<1 / 2$. Under this condition, $\delta^{*}(\mathbf{n})$ tends to zero for $s(\mathbf{n})=$ $O(1)$ as well, if we choose $a<1 / 2-d$, which we do.

Theorem 1.5. Suppose that $n_{1}<n_{2} \leq n_{1}^{3 / 2-d}$ and $n_{1}$ is sufficiently large. Then

$$
\mathrm{P}\left(\max _{\mathcal{M}}\left|\frac{R(\mathcal{M})}{n_{1}^{2} f(s(\mathbf{n}))}-1\right| \geq 1.01 \delta^{*}(\mathbf{n})\right) \leq \mathcal{P}(\mathbf{n}) .
$$


Notes. These two theorems together can be viewed as a quantified analogue of the AKL theorem. They effectively show how the width of an interval enclosing the scaled concentration point determines the probability that the full range of the corresponding total rank is contained in this interval. They demonstrate that while the likely bounds for the range of $Q(\mathcal{M})$ in the AKL theorem are rather sharp in the full range of $n_{1}, n_{2}$, those for $R(\mathcal{M})$ are sharp only in the extreme cases, namely $n_{2} / n_{1} \rightarrow 1$ and $n_{2} / n_{1} \rightarrow \infty$.

That we had to impose the constraint $n_{2} \leq n_{1}^{3 / 2-d}$ came as a surprise. Most likely, it is an artifact of our method, and we have no reason to doubt that only the condition $n_{2} / n_{1} \rightarrow \infty$ is needed for

$$
\max _{\mathcal{M}}\left|n_{1}^{-2} R(\mathcal{M})-\frac{1}{2}\right| \rightarrow 0
$$

in probability. For $n_{2} \gg n_{1}^{2}$, for instance, with probability

$$
\frac{\left(n_{2}\right)_{n_{1}}}{n_{2}^{n_{1}}} \sim \exp \left(-\frac{n_{1}^{2}}{2 n_{2}}\right) \rightarrow 1
$$

the best marriage candidates for $n_{1}$ men are all distinct. So w.h.p. in $n_{1}$ steps of the men-to-women proposal algorithm the men will propose to, and will be accepted by their respective best choices. By Theorem 1.3, w.h.p. this stable matching $\mathcal{M}_{1}$, with $Q\left(\mathcal{M}_{1}\right)=n_{1}$, is unique. As for $R\left(\mathcal{M}_{1}\right)$, it equals the sum of $n_{1}$ independent, $\left[n_{1}\right]$-Uniforms $U_{j}$, whence in probability

$$
n_{1}^{-2} R\left(\mathcal{M}_{1}\right)=n_{1}^{-2} \sum_{j=1}^{n_{1}} U_{j} \rightarrow \int_{0}^{1} x d x=\frac{1}{2} .
$$

It is possible though that appearance of the growth bound for $n_{2}$ in our argument signals an abrupt change in the likely structure of the already unique stable matching, when $n_{2}$ passes through the threshold $n_{1}^{3 / 2}$. Coincidentally, I learned from Yash Kanoria's e-mail that they also might have tacitly assumed that $n_{2}$ did not grow too fast with $n_{1}$.

Finally, we use the powerful result of Irving and Leather [8] on the lattice of stable matchings, in combination with an analogue of the formula for $P(\mathbf{n})$ in Lemma 1.2, to prove

Theorem 1.6. Let $m(\mathbf{n})$ and $w(\mathbf{n})$ stand for the fraction of men and for the fraction of women with more than one stable partner. If $n_{2}-n_{1} \geq$ $n_{1}^{1 / 2}\left(\log n_{1}\right)^{-\gamma},(\gamma<1)$, then $\lim m(\mathbf{n})=\lim w(\mathbf{n})=0$ in probability.

With some effort, the logarithmic factor (not the power of $n_{1}$ though) could be improved. Extension of our approach to the condition " $n_{2}-n_{1} \geq 1$ " in the AKL theorem is rather problematic. 


\section{Proof of Lemma 1.2}

First of all, it suffices to consider the injection $M$ such that $M(i)=i$ for all $i \in\left[n_{1}\right]$.

Introduce the pair of two $n_{1} \times n_{2}$ matrices $\mathbf{X}=\left\{X_{i, j}\right\}, \mathbf{Y}=\left\{Y_{i, j}\right\}$ with all $2 n_{1} n_{2}$ entries being independent, $[0,1]$-uniform random variables. Reading the entries of each row of $\mathbf{X}$ and of each column of $\mathbf{Y}$ in increasing order, we generate the independent, uniform preferences of each of $n_{1}$ men and each of $n_{2}$ women respectively

With probability $1, M$ is stable iff

$$
\begin{aligned}
& X_{r, s}>X_{r, r}, \quad \forall r \leq n_{1}, s>n_{1}, \\
& X_{r, s}>X_{r, r} \text { or } Y_{r, s}>Y_{r, r}, \quad \forall r, s \leq n_{1}, r \neq s .
\end{aligned}
$$

Call the corresponding events $B_{r, s}$. Crucially, conditioned on the values $x_{i}=$ $X_{i, i}, y_{j}=Y_{j, j}, i \leq n_{1}, j \leq n_{1}$, the $n_{1} n_{2}-n_{1}$ events $B_{r, s}$ are independent, and so the conditional probability that $M$ is stable equals

$$
\prod_{h \leq n_{1}<j_{1}}\left(1-x_{h}\right) \prod_{i, j \leq n_{1}, i \neq j}\left(1-x_{i} y_{j}\right)=\prod_{i, j \leq n_{1}, i \neq j}\left(1-x_{i} y_{j}\right) \prod_{h \leq n_{1}} \bar{x}_{h}^{n_{2}-n_{1}} .
$$

Integrating this expression over the cube $[0,1]^{2 n_{1}}$, i.e. using the Fubini theorem, we obtain the integral formula for $P(\mathbf{n})$. As for $P_{k}(\mathbf{n}), P_{k, \ell}(\mathbf{n})$ and $P_{k, \ell}(\mathbf{n})$, it suffices to consider $P_{k, \ell}(\mathbf{n})$. Indeed, the integral representation for $P_{k}(\mathbf{n})$ will follow by setting $\eta=1$ and dropping the $\left[\eta^{\ell-n_{1}}\right]$ (extraction) operator. (In fact the formula for $P(\mathbf{n})$ is similarly obtained by setting $\xi=1, \eta=1$ and dropping the $\left[\xi^{k-n_{1}} \eta^{\ell-n_{1}}\right]$ operator.)

Notice that the wives' and the husbands' total ranks are given by

$$
\begin{aligned}
& Q(M)=n_{1}+\sum_{i=1}^{n_{1}}\left|\left\{j \leq n_{2}: X_{i, j}<X_{i, i}\right\}\right|, \\
& R(M)=n_{1}+\sum_{j=1}^{n_{1}}\left|\left\{i \leq n_{1}: Y_{i, j}>Y_{j, j}\right\}\right| .
\end{aligned}
$$

Indeed, e.g. the number of women whom the man $i$ likes as much as he does his wife $i$ is 1 plus the number of women $j$ such that $X_{i, j}<X_{i, i}$, whence the formula for $Q(M)$. Of course, if $M$ is stable, all those women $j$ are among the first $n_{1}$ women. Our task is to compute the probability of the event $\{M$ is stable $\} \cap\{Q(M)=k, R(M)=\ell\}$. Let us determine $P_{k, \ell}(M \mid \mathbf{x}, \mathbf{y})$, the conditional probability of this event given $X_{i, i}=x_{i}, Y_{j, j}=y_{j},(1 \leq i, j \leq$ $\left.n_{1}\right)$. Once it is done, the unconditional $P_{k, \ell}(\mathbf{n})$ is obtained via integrating $P_{k, \ell}(M \mid \mathbf{x}, \mathbf{y})$ over the cube $[0,1]^{2 n_{1}}$.

To this end, we resort to the generating functions and write

$$
P_{k, \ell}(M \mid \mathbf{x}, \mathbf{y})=\left[\xi^{k} \eta^{\ell}\right] \mathrm{E}\left[1_{\{M \text { is stable }\}} \xi^{Q(M)} \eta^{R(M)} \mid \mathbf{x}, \mathbf{y}\right]
$$


Notice at once that on the event $\{M$ is stable $\}, Q(M)$ nominally dependent on the whole $\mathbf{x}$ is actually a function of $\left\{x_{i}\right\}_{i \leq n_{1}}$. To determine the underlying polynomial of $\xi, \eta$, it suffices to consider $\xi, \eta \in[0,1]$, in which case a probabilistic interpretation of this polynomial allows to speed up the otherwise clumsy derivation.

Go through the unmatched pairs $(i, j)$, i.e. $j \neq i$. Let $j \leq n_{1}$. Whenever $X_{i, j}>X_{i, i}\left(=x_{i}\right)$, mark $(i, j)$ with probability $\xi$; whenever $Y_{i, j}>Y_{j, j}(=$ $\left.y_{j}\right)$, color $(i, j)$ with probability $\eta$, independently of all the previous mark/ color operations. If both $X_{i, j}>X_{i, i}$ and $Y_{i, j}>Y_{j, j}$ we perform both mark and color operations on $(i, j)$, independently of each other. On the event $\{M$ is stable $\}$ no such pair exists, of course. Then

$$
\mathrm{E}\left[1_{\{M \text { is stable }\}} \xi^{Q(M)} \eta^{R(M)} \mid \mathbf{x}, \mathbf{y}\right]=\xi^{n} \eta^{n} \mathrm{P}(\mathcal{B} \mid \mathbf{x}, \mathbf{y}),
$$

where $\mathcal{B}$ is the event " $M$ is stable, and all pairs $(i, j)$ eligible for mark/color operation are marked/colored". Now

$$
\mathcal{B}=\left(\bigcap_{1 \leq i \neq j \leq n_{1}} \mathcal{B}_{i, j}\right) \bigcap\left(\bigcap_{r \leq n_{1}<s} B_{r, s}\right) ;
$$

here $B_{r, s}$ is defined in the first line of (2.1), and $\mathcal{B}_{i, j}$ is the event

$$
\begin{aligned}
& \left\{\left(X_{i, i}<X_{i, j}, Y_{j, j}<Y_{i, j}\right)\right. \\
& \sqcup\left(X_{i, i}<X_{i, j}, Y_{j, j}>Y_{i, j},(i, j) \text { is marked }\right) \\
& \left.\sqcup\left(X_{i, i}>X_{i, j}, Y_{j, j}<Y_{i, j},(i, j) \text { is colored }\right)\right\} .
\end{aligned}
$$

Conditioned on the event $\left\{X_{i, i}=x_{i}\right\}_{i \leq n_{1}} \cap\left\{Y_{j, j}=y_{j}\right\}_{j \leq n_{1}}$, the $\left(n_{1} n_{2}-n_{1}\right)$ events $\mathcal{B}_{i, j}, B_{r, s}$ are all independent, and

$$
\begin{aligned}
\mathrm{P}\left(\mathcal{B}_{i, j} \mid \mathbf{x}, \mathbf{y}\right) & =\left(1-x_{i}\right)\left(1-y_{j}\right)+\xi\left(1-x_{i}\right) y_{j}+\eta x_{i}\left(1-y_{j}\right) \\
& =\bar{x}_{i} \bar{y}_{j}+\xi \bar{x}_{i} y_{j}+\eta x_{i} \bar{y}_{j}, \\
\mathrm{P}\left(B_{r, s} \mid \mathbf{x}, \mathbf{y}\right) & =1-x_{r}=\bar{x}_{r} .
\end{aligned}
$$

Collecting the pieces, and integrating over $(\mathbf{x}, \mathbf{y}) \in[0,1]^{2 n_{1}}$, we obtain the desired formula for $P_{k, \ell}(\mathbf{n})$.

\section{Proof of Theorem 1.3}

To estimate sharply the $2 n_{1}$-dimensional integral representing $P(\mathbf{n})$ in Lemma 1.2, we will use the following facts collected and proved in [13]. Let $X_{1}, \ldots, X_{n}$ be independent, $[0,1]$-uniform random variables. Denote

$$
\mathcal{S}_{n}=\sum_{j=1}^{n} X_{j}, \quad \mathcal{T}_{n}=\frac{\sum_{j=1}^{n} X_{j}^{2}}{\mathcal{S}_{n}^{2}} .
$$


Also, let $L_{1}, \ldots, L_{n}$ denote the lengths of the $n$ consecutive subintervals of $[0,1]$ obtained by independently selecting $n-1$ points, each uniformly distributed on $[0,1]$; in particular, $\sum_{j} L_{j}=1$. Define $T_{n}=\sum_{j} L_{j}^{2}, L_{n}^{+}=$ $\max _{j} L_{j}$.

Lemma 3.1. Let $f_{n}(s), f_{n}(s, t), g_{n}(t)$ denote the density of $\mathcal{S}_{n},\left(\mathcal{S}_{n}, \mathcal{T}_{n}\right)$ and $T_{n}$ respectively. Then

$$
f_{n}(s)=\frac{s^{n-1}}{(n-1) !} \mathrm{P}\left(L_{n}^{+} \leq s^{-1}\right)
$$

in particular

$$
f_{n}(s) \leq \frac{s^{n-1}}{(n-1) !}
$$

Furthermore,

$$
f_{n}(s, t) \leq \frac{s^{n-1}}{(n-1) !} g_{n}(t)
$$

We will also need

Lemma 3.2. (1) In probability,

$$
\lim _{n \rightarrow \infty} \frac{L_{n}^{+}}{n^{-1} \log n}=1, \quad \lim _{n \rightarrow \infty} n T_{n}=2,
$$

and (2)

$$
\mathrm{P}\left(n T_{n} \geq 3\right)=O\left(n^{-1}\right) .
$$

The relation (3.1) can be found in Feller [3], Ch. 1, for instance, but the inequality (3.3) was new. Both of these relations were proved in [13 by using the fact that the joint density of $L_{1}, \ldots, L_{n-1}$ is $(n-1)$ ! whenever the density is positive. As for Lemma 3.2, (1), its proof was based on a classic equidistribution of $\left(L_{1}, \ldots, L_{n}\right)$ and $\left\{W_{j} / \sum_{k} W_{k}: 1 \leq j \leq n\right\}$, where $W_{j}$ are independent exponentials with parameter 1 , see 13 for the references. This equidistribution delivers the part (2) as follows. Observe that $\mathrm{E}[W]=1, \mathrm{E}\left[W^{2}\right]=2$. Choose $a>2$ and $b<1$ such that $a / b^{2}<3$; for instance $a=2+1 / 7$ and $b=1-1 / 7$. Then, denoting $\mathcal{W}^{(\ell)}=\sum_{j} W_{j}^{\ell}$,

$$
\begin{aligned}
\mathrm{P}\left(n T_{n} \geq 3\right) & =\mathrm{P}\left(\frac{\mathcal{W}^{(2)}}{\left(\mathcal{W}^{(1)}\right)^{2}} \geq \frac{3}{n}\right) \leq \mathrm{P}\left(\mathcal{W}^{(2)} \geq \text { an or } \mathcal{W}^{(1)}<b n\right) \\
& \leq \mathrm{P}\left(\mathcal{W}^{(2)} \geq a n\right)+\mathrm{P}\left(W^{(1)}<b n\right) .
\end{aligned}
$$

By Chebyshev's inequality, each of these probabilities is of order $O\left(n^{-1}\right)$, and then so is $\mathrm{P}\left(n T_{n} \geq 3\right)$, which proves the part (2). (We note in passing that this probability is, in fact, much smaller, certainly below $\exp \left(-c n^{1 / 3}\right)$.) 
3.1. Upper bound for $P(\mathbf{n})$. To bound $P(\mathbf{n})$ from above, we evaluate, asymptotically, the $2 n_{1}$-dimensional integral in Lemma 1.2, integrating first over $\mathbf{y}$, and second over $\mathbf{x}$. Introduce $s=\sum_{i} x_{i}, t=\sum_{i} x_{i}^{2}$, and $s_{j}=$ $\sum_{i \neq j} x_{i}=s-x_{j}, t_{j}=\sum_{i \neq j} x_{i}^{2}$. Let $\int_{1}, \int_{2}$ denote the contribution to $P(\mathbf{n})$ coming from $(\mathbf{x}, \mathbf{y})$ with $\frac{t}{s^{2}} \geq 3 / n_{1}$, and with $\frac{t}{s^{2}} \leq 3 / n_{1}$ respectively. Since $1-\alpha \leq e^{-\alpha}$, we have

$$
\begin{aligned}
\int_{1} & \leq \overbrace{\int_{\frac{t}{t^{2}} \geq 3 / n_{1}}^{n_{1}}}^{n_{1}}\left(\prod_{j} \int_{0}^{1} e^{-y s_{j}} d y\right) e^{-\left(n_{2}-n_{1}\right) s} d \mathbf{x} \\
& =\overbrace{\int_{\frac{t}{s^{2}} \geq 3 / n_{1}}^{n_{1}} \ldots \int}\left(\prod_{j} \frac{1-e^{-s_{j}}}{s_{j}}\right) \cdot e^{-\left(n_{2}-n_{1}\right) s} d \mathbf{x} .
\end{aligned}
$$

Now it is easy to check that

$$
\left(\log \frac{1-e^{-\xi}}{\xi}\right)^{\prime} \geq-\min \left\{\frac{1}{2}, \frac{1}{\xi}\right\}
$$

therefore

$$
\sum_{j} \log \frac{1-e^{-s_{j}}}{s_{j}} \leq n_{1} \log \frac{1-e^{-s}}{s}+2, \quad \forall s>0 .
$$

Applying Lemma 3.1, (3.3), we obtain then

$$
\begin{aligned}
\int_{1} & \leq e^{2} \frac{\mathrm{P}\left(T_{n_{1}} \geq 3 / n_{1}\right)}{\left(n_{1}-1\right) !} \int_{0}^{\infty} s^{-1} I(s) d s, \\
I(s) & :=e^{-\left(n_{2}-n_{1}\right) s}\left(1-e^{-s}\right)^{n_{1}} .
\end{aligned}
$$

By Lemma 3.2, (2), the front probability is $O\left(n_{1}^{-1}\right)$, at most. In addition

$$
\begin{aligned}
& \int_{0}^{\infty} s^{-1} I(s) d s \leq \int_{0}^{\infty} e^{-\left(n_{2}-n_{1}\right) s}\left(1-e^{-s}\right)^{n_{1}-1} d s \\
& =\int_{0}^{1} z^{n_{2}-n_{1}-1}(1-z)^{n_{1}-1} d z=\frac{1}{\left(n_{2}-1\right)\left(\begin{array}{l}
n_{2}-2 \\
n_{1}-1
\end{array}\right)} \\
& =\frac{n_{2}}{n_{1}} \cdot \frac{1}{\left(n_{2}-n_{1}\right)\left(\begin{array}{l}
n_{2} \\
n_{1}
\end{array}\right)} .
\end{aligned}
$$

Therefore

$$
\int_{1} \leq_{b} \frac{1}{n_{1} n_{1} !} \cdot \frac{1}{\left(n_{2}-n_{1}\right)\left(\begin{array}{c}
n_{2} \\
n_{1}
\end{array}\right)} .
$$

(We use $F_{n} \leq_{b} G_{n}$ to indicate that $F_{n}=O\left(G_{n}\right)$ when the expression for $G_{n}$ is too bulky.) 
Turn to $\int_{2}$, i.e. the contribution to $P(\mathbf{n})$ from $\mathbf{x}$ with $t s^{-2}<3 n^{-1}$. Using $1-\alpha \leq e^{-\alpha-\alpha^{2} / 2}$ this time, we need to bound, sharply,

$$
\prod_{j} \int_{0}^{1} \exp \left(-y s_{j}-y^{2} t_{j} / 2\right) d y
$$

Introduce a new variable $z=y s_{j}$ in the $j$-th factor of the product. Using $s_{j} \leq s, t_{j} \leq t$, where appropriate, and integrating by parts once, we have

$$
\begin{gathered}
\int_{0}^{1} \exp \left(-y s_{j}-\frac{y^{2} t_{j}}{2}\right) d y \leq \frac{1}{s_{j}} \int_{0}^{s_{j}} \exp \left(-z-z^{2} \frac{t_{j}}{2 s^{2}}\right) d z \\
\leq \frac{1}{s_{j}}\left(\int_{0}^{s} \exp \left(-z-z^{2} \frac{t_{j}}{2 s^{2}}\right) d z-\left(s-s_{j}\right) e^{-s-t / 2}\right) \\
=\frac{1}{s_{j}}\left(1-e^{-s-t_{j} / 2}-\frac{t_{j}}{s^{2}} \int_{0}^{s} z \exp \left(-z-z^{2} \frac{t_{j}}{2 s^{2}}\right) d z-x_{j} e^{-s-t / 2}\right) \\
\leq \frac{1-e^{-s-t / 2}}{s_{j}}\left(1-\frac{\frac{t_{j}}{s^{2}}}{1-e^{-s-t / 2}} \int_{0}^{s} z \exp \left(-z-z^{2} \frac{t_{j}}{2 s^{2}}\right) d z-\frac{x_{j}}{e^{s+t / 2}-1}\right) \\
\leq \frac{1-e^{-s-t / 2}}{s_{j}}\left(1-\frac{t_{j}}{s^{2}} F\left(s, \frac{t}{s^{2}}\right)-\frac{x_{j}}{e^{s+t / 2}-1}\right) \\
\leq \frac{1-e^{-s-t / 2}}{s_{j}} \exp \left(-\frac{t_{j}}{s^{2}} F\left(s, \frac{t}{s^{2}}\right)-\frac{x_{j}}{e^{s+t / 2}-1}\right)
\end{gathered}
$$

where

$$
F(s, u):=\frac{1}{1-\exp \left(-s-s^{2} \frac{u}{2}\right)} \int_{0}^{s} z \exp \left(-z-z^{2} \frac{u}{2}\right) d z
$$

in particular,

$$
F(s, 0)=1-\frac{s}{e^{s}-1}, \quad F(s, u)=F(s, 0)(1+O(u)), \quad u \downarrow 0,
$$

uniformly for $s>0$. Next,

$$
\begin{aligned}
1-e^{-s-t / 2} & =\left(1-e^{-s}\right)\left(1+\frac{1-e^{-t / 2}}{e^{s}-1}\right) \leq\left(1-e^{-s}\right)\left(1+\frac{t / 2}{e^{s}-1}\right) \\
& \leq\left(1-e^{-s}\right) \exp \left(\frac{s^{2}}{2\left(e^{s}-1\right)} \frac{t}{s^{2}}\right)
\end{aligned}
$$

so that

$$
\left(1-e^{-s-t / 2}\right)^{n_{1}} \leq\left(1-e^{-s}\right)^{n_{1}} \exp \left(\frac{s^{2}}{2\left(e^{s}-1\right)} \frac{n_{1} t}{s^{2}}\right)
$$


with the last exponent bounded as $n_{1} \rightarrow \infty$, uniformly for all $\mathbf{x}$ in question, i.e. meeting the constraint $\frac{n_{1} t}{s^{2}} \leq 3$. Also, as $\sum_{j} x_{j}=s$,

$$
\begin{aligned}
\prod_{j} s_{j} & =s^{n_{1}} \prod_{j}\left(1-\frac{x_{j}}{s}\right)=s^{n_{1}} \frac{\prod_{j^{\prime}}\left(1-\frac{x_{j^{\prime}}^{2}}{s^{2}}\right)}{\prod_{j}\left(1+\frac{x_{j}}{s}\right)} \\
& \geq e^{-1} s^{n_{1}}\left(1-\frac{t}{s^{2}}\right) \geq e^{-1} s^{n_{1}}\left(1-\frac{3}{n_{1}}\right) .
\end{aligned}
$$

Collecting the bounds, and using $\sum_{j} t_{j}=\left(n_{1}-1\right) t$, we get

$$
\begin{aligned}
& \text { 3.11) } \prod_{j} \int_{0}^{1} \exp \left(-y s_{j}-\frac{y^{2} t_{j}}{2}\right) d y \\
& \lesssim e\left(\frac{1-e^{-s}}{s}\right)^{n_{1}} \exp \left(-\frac{\left(n_{1}-1\right) t}{s^{2}} F(s, 0)-\frac{s}{e^{s+t / 2}-1}+\frac{s^{2}}{2\left(e^{s}-1\right)} \frac{n_{1} t}{s^{2}}\right) .
\end{aligned}
$$

(We use $F_{n} \lesssim G_{n}$ to mean that $\lim \sup F_{n} / G_{n} \leq 1$.) And for the remaining factor $\prod_{h} \bar{x}_{h}$ in the integrand for $P(\mathbf{n})$ we have

$$
\begin{aligned}
\prod_{h} \bar{x}_{h}^{n_{2}-n_{1}} & \leq \exp \left(-\left(n_{2}-n_{1}\right) \sum_{h} x_{h}-\frac{n_{2}-n_{1}}{2} \sum_{h} x_{h}^{2}\right) \\
& =\exp \left(-\left(n_{2}-n_{1}\right) s-\frac{\left(n_{2}-n_{1}\right) s^{2}}{2} \frac{t}{s^{2}}\right) .
\end{aligned}
$$

Now, $s$ and $t$ are the generic values of the random variables $\mathcal{S}_{n_{1}}=\sum_{j} X_{j}$ and $\sum_{j} X_{j}^{2}$ respectively. By (3.3), the joint density of $\mathcal{S}_{n_{1}}$ and $\mathcal{T}_{n_{1}}=\mathcal{S}_{n_{1}}^{-2} \sum_{j} X_{j}^{2}$ is bounded above by $\frac{s^{n_{1}-1}}{\left(n_{1}-1\right) !}$ times the density of $T_{n_{1}}=\sum_{j} L_{j}^{2}$. So integrating the product of the bounds in (3.11) and (3.12) over $\mathrm{x} \in[0,1]^{n_{1}}$, meeting the constraint $\frac{t}{s^{2}} \leq 3$, we obtain

$$
\begin{gathered}
\int_{2} \leq \frac{e}{\left(n_{1}-1\right) !} \int_{0}^{n_{1}} \frac{I(s)}{s} \mathrm{E}\left[U_{n_{1}}(s)\right] d s \\
U_{n_{1}}(s):=1_{\left\{T_{n_{1}} \leq \frac{3}{n_{1}}\right\}} \exp \left(-\frac{\left(n_{2}-n_{1}\right) s^{2}}{2 n_{1}} n_{1} T_{n_{1}}\right. \\
\left.-\left(n_{1}-1\right) T_{n_{1}} F(s, 0)-\frac{s}{\exp \left(s+s^{2} T_{n_{1}} / 2\right)-1}+\frac{s^{2}}{2\left(e^{s}-1\right)} n_{1} T_{n_{1}}\right) ;
\end{gathered}
$$

recall that $I(s)$ was defined in (3.5).

The function $I(s)$ attains its maximum at $s(\mathbf{n})=\log \frac{n_{2}}{n_{2}-n_{1}}$. We anticipate, but will have to prove, that the dominant contribution to the integral in (3.13) comes from

$$
s \in \mathcal{I}(\mathbf{n}):=[(1-\delta(\mathbf{n})) s(\mathbf{n}),(1+\delta(\mathbf{n})) s(\mathbf{n})],
$$


for some $\delta(\mathbf{n}) \rightarrow 0$.

To this end, we need to have a close look at $\mathrm{E}\left[U_{n_{1}}(s)\right]$. First, throwing out the negative summands from the exponent in the expression (3.13) for $U_{n_{1}}(s)$, we have

$$
\begin{aligned}
U_{n_{1}}(s) & \leq 1_{\left\{T_{n_{1}} \leq \frac{3}{n_{1}}\right\}} \cdot \sup _{s>0} \exp \left(\frac{s^{2}}{2\left(e^{s}-1\right)} n_{1} T_{n_{1}}\right) \\
& \leq \sup _{s>0} \exp \left(\frac{3 s^{2}}{2\left(e^{s}-1\right)}\right)<\infty .
\end{aligned}
$$

Consider $s \in \mathcal{I}(\mathbf{n})$. For the first term in the exponent for $U_{n_{1}}(s)$,

$$
\begin{aligned}
\frac{\left(n_{2}-n_{1}\right) s^{2}}{2} T_{n_{1}} & =\frac{\left(n_{2}-n_{1}\right) s^{2}(\mathbf{n})}{2 n_{1}} n_{1} T_{n_{1}}+n_{1} T_{n_{1}} O\left(\delta(\mathbf{n}) \frac{s^{2}(\mathbf{n})\left(n_{2}-n_{1}\right)}{n_{1}}\right) \\
& =\frac{\left(n_{2}-n_{1}\right) s^{2}(\mathbf{n})}{2 n_{1}}\left(n_{1} T_{n_{1}}\right)+o(1),
\end{aligned}
$$

because

$$
\frac{s^{2}(\mathbf{n})\left(n_{2}-n_{1}\right)}{n_{1}}=\frac{\left(n_{2}-n_{1}\right) \log ^{2} \frac{n_{2}}{n_{2}-n_{1}}}{n_{1}}=O(1)
$$

for $n_{2}>n_{1} \rightarrow \infty$ and $n_{1} T_{n_{1}} \leq 3$. Furthermore

$$
F(s, 0)=F(s(\mathbf{n}), 0)+O\left(\delta(\mathbf{n}) s(\mathbf{n}) e^{-s(\mathbf{n})(1-\delta(\mathbf{n}))}\right)=F(s(\mathbf{n}), 0)+o(1) .
$$

For $T_{n_{1}} \leq \frac{3}{n_{1}}$, two remaining terms in the exponent for $U_{n_{1}}(s)$ are bounded for all $s>0$ as well. So, using $n_{1} T_{n_{1}} \rightarrow 2$ in probability, by the bounded convergence theorem, we obtain: uniformly for $s \in \mathcal{I}(\mathbf{n})$,

$$
\begin{aligned}
\lim \mathrm{E}\left[U_{n_{1}}(s)\right]=\exp (-\lim & \frac{\left(n_{2}-n_{1}\right) s(\mathbf{n})^{2}}{n_{1}}-2 \lim F(s(\mathbf{n}), 0) \\
& \left.+\lim \frac{s^{2}(\mathbf{n})-s(\mathbf{n})}{e^{s(\mathbf{n})}-1}\right),
\end{aligned}
$$

where $n_{2}>n_{1} \rightarrow \infty$ in such a way that all three limits on the RHS exist. Notice that, since $s(\mathbf{n})=\log \frac{n_{2}}{n_{2}-n_{1}}$, the limits are bounded by absolute constants whether $\lim \sup s(\mathbf{n})$ is finite or infinite. Effectively this means that $\mathrm{E}\left[U_{n_{1}}(s)\right] \sim e^{-h(\mathbf{n})}$, uniformly for $s \in \mathcal{I}(\mathbf{n})$, where, by (3.9),

$$
\begin{aligned}
h(\mathbf{n}) & :=\frac{\left(n_{2}-n_{1}\right) s^{2}(\mathbf{n})}{n_{1}}+2 F(s(\mathbf{n}), 0)+\frac{s(\mathbf{n})-s^{2}(\mathbf{n})}{\exp (s(\mathbf{n}))-1} \\
& =2+\frac{\left(n_{2}-n_{1}\right) s^{2}(\mathbf{n})}{n_{1}}-\frac{s(\mathbf{n})+s^{2}(\mathbf{n})}{e^{s(\mathbf{n})}-1} \\
& =2-\frac{s(\mathbf{n})}{e^{s(\mathbf{n})}-1} .
\end{aligned}
$$


provided that $n_{2}>n_{1} \rightarrow \infty$. Consequently

$$
\int_{s \in \mathcal{I}(\mathbf{n})} \frac{I(s)}{s} \mathrm{E}\left[U_{n_{1}}(s)\right] d s \sim \frac{e^{-h(\mathbf{n})}}{s(\mathbf{n})} \int_{s \in \mathcal{I}(\mathbf{n})} I(s) d s .
$$

The next step is to evaluate, asymptotically, the integral in (3.16).

Substituting $z=e^{-s}$, we rewrite

$$
\begin{aligned}
\int_{s \in \mathcal{I}(\mathbf{n})} I(s) d s & =\int_{z \in\left[z_{1}, z_{2}\right]} K(z) d z, \quad K(z):=z^{n_{2}-n_{1}-1}(1-z)^{n_{1}}, \\
z_{1,2} & :=\left(\frac{n_{2}-n_{1}}{n_{2}}\right)^{1 \pm \delta(\mathbf{n})} .
\end{aligned}
$$

Now

$$
\int_{z \in[0,1]} K(z) d z=\frac{1}{\left(n_{2}-n_{1}\right)\left(\begin{array}{l}
n_{2} \\
n_{1}
\end{array}\right)},
$$

and we need to bound the contributions of the two tail integrals, over $\left[0, z_{1}\right]$ and $\left[z_{2}, 1\right]$.

Consider first the case $n_{2}-n_{1} \ll n_{2}$, i.e. $s(\mathbf{n}) \rightarrow \infty$. We have

$$
\begin{aligned}
\int_{z \in\left[0, z_{1}\right]} K(z) d z & \leq \int_{z \in\left[0, z_{1}\right]} z^{n_{2}-n_{1}-1} d z \\
& =\frac{z_{1}^{n_{2}-n_{1}}}{n_{2}-n_{1}}=\left(\frac{n_{2}-n_{1}}{n_{2}}\right)^{(1+\delta(\mathbf{n}))\left(n_{2}-n_{1}\right)} .
\end{aligned}
$$

So, using (3.17) and $\left(\begin{array}{l}b \\ a\end{array}\right) \leq\left(\frac{e b}{a}\right)^{a}, m^{1 / m} \leq e^{1 / e}$, we have

$$
\frac{\int_{0}^{z_{1}} K(z) d z}{\int_{0}^{1} K(z) d z} \leq \varepsilon_{1}(\mathbf{n}):=\exp \left(-0.99 \delta(\mathbf{n}) s(\mathbf{n})\left(n_{2}-n_{1}\right)\right) \rightarrow 0,
$$

if $\delta(\mathbf{n})=s^{-b}(\mathbf{n}), b<1 ;(\delta(\mathbf{n}) \rightarrow 0$ obviously $)$. Next, since for such $\delta(\mathbf{n})$

$$
n_{1} z_{2}=n_{1}\left(\frac{n_{2}-n_{1}}{n_{2}}\right)^{1-\delta(\mathbf{n})}=\frac{n_{1}\left(n_{2}-n_{1}\right)}{n_{2}}\left(\frac{n_{2}}{n_{2}-n_{1}}\right)^{\delta(\mathbf{n})} \rightarrow \infty,
$$

we bound

$$
\begin{aligned}
\int_{z_{2}}^{1} K(z) d z & \leq \int_{z_{2}}^{\infty} z^{n_{2}-n_{1}-1} e^{-n_{1} z} d z \leq \frac{1}{n_{1}^{n_{2}-n_{1}}} \int_{n_{1} z_{2}}^{\infty} y^{n_{2}-n_{1}} e^{-y} d y \\
& =O\left(z_{2}^{n_{2}-n_{1}} e^{-n_{1} z_{2}}\right) ;
\end{aligned}
$$

the last estimate follows from

$$
\max _{y \geq n_{1} z_{2}} \frac{d \log \left(y^{n_{2}-n_{1}} e^{-y}\right)}{d y}=-1+\frac{n_{2}-n_{1}}{n_{1} z_{2}} \rightarrow-1 .
$$


It follows easily that

$$
\begin{aligned}
\frac{\int_{z_{2}}^{1} K(z) d z}{\int_{0}^{1} K(z) d z} & \leq \exp \left(-0.99\left(n_{2}-n_{1}\right)\left(\frac{n_{2}}{n_{2}-n_{1}}\right)^{\delta(\mathbf{n})}\right) \\
& =\exp \left(-0.99\left(n_{2}-n_{1}\right) e^{\delta(\mathbf{n}) s(\mathbf{n})}\right)=: \varepsilon_{2}(\mathbf{n}) .
\end{aligned}
$$

By (3.19) and (3.20), if $\delta(\mathbf{n}) s(\mathbf{n}) \rightarrow \infty$ then

$$
\frac{\int_{z \notin\left[z_{1}, z_{2}\right]} K(z) d z}{\int_{0}^{1} K(z) d z} \leq \varepsilon(\mathbf{n}):=\max \left\{\varepsilon_{1}(\mathbf{n}), \varepsilon_{2}(\mathbf{n})\right\}=\varepsilon_{1}(\mathbf{n}) \rightarrow 0 .
$$

So, by (3.17), for $n_{2} \gg n_{2}-n_{1}$ we have

$$
\int_{s \in \mathcal{I}(\mathbf{n})} I(s) d s=\int_{z \in\left[z_{1}, z_{2}\right]} K(z) d z \sim \int_{z \in[0,1]} K(z) d z=\frac{1}{\left(n_{2}-n_{1}\right)\left(\begin{array}{l}
n_{2} \\
n_{1}
\end{array}\right)},
$$

and the relative contribution of the tail $s \notin \mathcal{I}(\mathbf{n})$ is of order $\varepsilon(\mathbf{n})$.

Let us prove that, for an appropriate $\delta(\mathbf{n}) \rightarrow 0$, (1) the equation (3.22) holds also when $n_{2}-n_{1}=\theta\left(n_{2}\right)$ and (2) the relative weight of $s \notin \mathcal{I}(\mathbf{n})$ is at most some alternative $\varepsilon(\mathbf{n}) \rightarrow 0$. The integrand $K(z)$ attains its maximum at $z^{*}=\frac{n_{2}-n_{1}-1}{n_{2}-1}$, and

$$
K\left(z^{*}\right)=\frac{\left(n_{2}-n_{1}-1\right)^{n_{2}-n_{1}-1} n_{1}^{n_{1}}}{\left(n_{2}-1\right)^{n_{2}-1}}=O\left(n_{1}^{-1 / 2}\left(\begin{array}{c}
n_{2} \\
n_{1}
\end{array}\right)^{-1}\right) .
$$

Furthermore

$$
\begin{aligned}
z_{1,2}-z^{*} & =\left(\frac{n_{2}-n_{1}}{n_{2}}\right)^{1 \pm \delta}-\frac{n_{2}-n_{1}-1}{n_{2}-1} \\
& =\mp \theta\left(\delta n_{1} / n_{2}\right)\left(1+O\left(\left(\delta n_{1}\right)^{-1}+\delta n_{1} / n_{2}\right)\right)
\end{aligned}
$$

the remainder term is $o(1)$ if, in addition to $\delta(\mathbf{n}) \rightarrow 0$, we impose the condition $\delta(\mathbf{n}) n_{1} \rightarrow \infty$. Simple calculus shows then that

$$
\log K\left(z_{1,2}\right)=\log K\left(z^{*}\right)-\theta\left(\delta^{2} n_{1}\right),\left.\quad \frac{d \log K(z)}{d z}\right|_{z=z_{1,2}}= \pm \theta\left(\delta n_{2}\right) .
$$

Since $\log K(z)$ is concave, we have then

$$
\begin{array}{ll}
\log K(z) \leq \log K\left(z_{1}\right)+\theta\left(\delta n_{2}\right)\left(z-z_{1}\right), & z \leq z_{1}, \\
\log K(z) \leq \log K\left(z_{2}\right)-\theta\left(\delta n_{2}\right)\left(z-z_{2}\right), & z \geq z_{2} .
\end{array}
$$

Therefore

$$
\int_{z \notin\left[z_{1}, z_{2}\right]} K(z) d z \leq K\left(z^{*}\right) \frac{\exp \left(-\theta\left(\delta^{2}(\mathbf{n}) n_{1}\right)\right)}{\theta\left(\delta(\mathbf{n}) n_{2}\right)} .
$$


BORIS PITTEL

Using (3.23) and (3.24), we see that the ratio of the upper bound above to the integral in (3.17) is of order

$$
\varepsilon(\mathbf{n}):=\frac{\exp \left(-\theta\left(\delta^{2}(\mathbf{n}) n_{1}\right)\right)}{\delta(\mathbf{n}) n_{1}^{1 / 2}}
$$

which tends to zero if, for instance, $\delta(\mathbf{n})=n_{1}^{-a}, a<1 / 2$. So indeed the equation (3.22) holds for $\delta(\mathbf{n})=n_{1}^{-a}, a<1 / 2$, and the relative contribution of the tail $s \notin \mathcal{I}(\mathbf{n})$ is at most this $\varepsilon(\mathbf{n})$.

Invoking (3.16), we see that if $n_{2}>n_{1} \rightarrow \infty$, then for $\mathcal{I}(\mathbf{n})$ defined in (3.14)

$$
\int_{s \in \mathcal{I}(\mathbf{n})} \frac{I(s)}{s} \mathrm{E}\left[U_{n_{1}}(s)\right] d s \sim \frac{e^{-h(\mathbf{n})}}{s(\mathbf{n})\left(n_{2}-n_{1}\right)\left(\begin{array}{l}
n_{2} \\
n_{1}
\end{array}\right)},
$$

if, picking $a<1 / 2$ and $b<1$, we define

$$
\delta(\mathbf{n}):= \begin{cases}s(\mathbf{n})^{-b}, & \text { if } s(\mathbf{n}) \rightarrow \infty, \\ n_{1}^{-a}, & \text { if } s(\mathbf{n})=O(1) .\end{cases}
$$

Finally, since $\mathrm{E}\left[U_{n_{1}}(s)\right]=O(1)$ uniformly for $s>0$, and $\frac{1-e^{-s}}{s}<1$, we get

$$
\int_{s \notin \mathcal{I}(\mathbf{n})} \frac{I(s)}{s} \mathrm{E}\left[U_{n_{1}}(s)\right] d s \leq_{b} \int_{z \notin\left[z_{1}, z_{2}\right]} z^{n_{2}-n_{1}-1}(1-z)^{n_{1}-1} d z .
$$

Like the integrals of $K(z)$, (for $\varepsilon(\mathbf{n})$ defined, correspondingly, for $n_{2} \gg$ $n_{2}-n_{1}$ and $\left.n_{2}-n_{1}=\theta\left(n_{2}\right)\right)$, the RHS integral is of order

$$
\begin{aligned}
& \varepsilon(\mathbf{n}) \int_{0}^{1} z^{n_{2}-n_{1}-1}(1-z)^{n_{1}-1} d z=\varepsilon(\mathbf{n}) \cdot \frac{n_{2} / n_{1}}{\left(n_{2}-n_{1}\right)\left(\begin{array}{l}
n_{2} \\
n_{1}
\end{array}\right)} \\
& \leq_{b} \varepsilon(\mathbf{n}) \frac{n_{2}}{n_{1}} \log \frac{n_{2}}{n_{2}-n_{1}} \int_{s \in \mathcal{I}(\mathbf{n})} \frac{I(s)}{s} \mathrm{E}\left[U_{n_{1}}(s)\right] d s .
\end{aligned}
$$

For $n_{2} \gg n_{2}-n_{1}$, i.e. $s(\mathbf{n}) \rightarrow \infty$, the outside factor is asymptotic to $\varepsilon(\mathbf{n}) s(\mathbf{n})$. So, by definition of $\varepsilon(\mathbf{n})$ in (3.24), and $\delta(\mathbf{n})$ in (3.27), this factor is of order

$$
s(\mathbf{n}) \exp \left(-0.5 s(\mathbf{n})^{1-b}\right)=\exp \left(-\theta\left(s(\mathbf{n})^{1-b}\right)\right) .
$$

For $n_{2}-n_{1}=\theta\left(n_{2}\right)$, by definition of $\varepsilon(\mathbf{n})$ in (3.25), and $\delta(\mathbf{n})$ in (3.27), the factor is of order

$$
\varepsilon(\mathbf{n})=\exp \left(-\theta\left(n_{1}^{1-2 a}\right)\right) .
$$

We conclude that if $n_{2}>n_{1} \rightarrow \infty$ then the bound (3.13) becomes

$$
\int_{2} \lesssim \frac{e^{1-h(\mathbf{n})}}{\left(n_{1}-1\right) !\left(n_{2}-n_{1}\right) s(\mathbf{n})}\left(\begin{array}{c}
n_{2} \\
n_{1}
\end{array}\right)^{-1},
$$


where $s(\mathbf{n})=\log \frac{n_{2}}{n_{2}-n_{1}}$, and $h(\mathbf{n})$ is defined in (3.15). In particular

$$
1-h(n)=-\frac{e^{s(\mathbf{n})}-1-s(\mathbf{n})}{e^{s(\mathbf{n})}-1} .
$$

Combining (3.31) and (3.7), and using $s(\mathbf{n})=O\left(\log n_{1}\right)$, we obtain

$$
P(\mathbf{n})=\int_{1}+\int_{2} \lesssim \frac{e^{1-h(\mathbf{n})}}{\left(n_{1}-1\right) !\left(n_{2}-n_{1}\right) s(\mathbf{n})}\left(\begin{array}{c}
n_{2} \\
n_{1}
\end{array}\right)^{-1} .
$$

Moreover, the contribution to $P(\mathbf{n})$ coming from $s \notin \mathcal{I}(\mathbf{n})$ and scaled by the RHS in (3.32) is of order given by (3.29) and (3.30) for $n_{2} \gg n_{2}-n_{1}$ and $n_{2} \gg n_{2}-n_{1}$, respectively.

Note. Observe that for $n_{2} / n_{1} \rightarrow \infty$ we have $s(\mathbf{n}) \sim \frac{n_{1}}{n_{2}} \rightarrow 0$, so $h(\mathbf{n}) \rightarrow 1$, and

$$
P(\mathbf{n}) \lesssim\left(\left(\begin{array}{l}
n_{2} \\
n_{1}
\end{array}\right) n_{1} !\right)^{-1}
$$

meaning that

$$
\mathrm{E}[S(\mathbf{n})]=\left(\begin{array}{l}
n_{2} \\
n_{1}
\end{array}\right) n_{1} ! \cdot P(\mathbf{n}) \lesssim 1 .
$$

Since $S(\mathbf{n}) \geq 1$, we see that $\mathrm{P}(S(\mathbf{n})=1) \rightarrow 1$.

3.2. Lower bound for $P(\mathbf{n})$. It remains to prove a matching lower bound for $P(\mathbf{n})$. For $\varepsilon>0$, let $D=D(\varepsilon)$ be a set of $\mathbf{x}=\left(x_{1}, \ldots, x_{n_{1}}\right) \geq \mathbf{0}$ defined by the constraints

$$
\begin{aligned}
& s(\mathbf{n})(1-\delta(\mathbf{n})) \leq s \leq s(\mathbf{n})(1+\delta(\mathbf{n})), \quad s:=\sum_{j=1}^{n_{1}} x_{j}, \\
& s^{-1} x_{j} \leq(1+\varepsilon) \frac{\log n_{1}}{n_{1}}, \quad 1 \leq j \leq n_{1}, \\
& (1-\varepsilon) \frac{2}{n_{1}} \leq s^{-2} t \leq(1+\varepsilon) \frac{2}{n_{1}}, \quad t:=\sum_{j=1}^{n_{1}} x_{j}^{2} .
\end{aligned}
$$

Here $\delta(\mathbf{n})$ is defined in (3.27); so the constraint (3.33) can be stated as $s \in \mathcal{I}(\mathbf{n})$, see (3.14). As $s(\mathbf{n})=O\left(\log n_{1}\right)$, the constraints (3.33), (3.34) imply that

$$
\max _{j} x_{j} \leq(1+\varepsilon) \frac{2 s(\mathbf{n}) \log n_{1}}{n_{1}}=O\left(\sigma_{n_{1}}\right), \quad \sigma_{n_{1}}:=n_{1}^{-1} \log ^{2} n_{1} .
$$

Since $\sigma_{n_{1}} \rightarrow 0, D$ is a subset of the cube $[0,1]^{n_{1}}$ for $n_{1}$ large enough. Likewise the constraints (3.33) and (3.35) imply that

$$
t=O\left(n_{1}^{-1} \log ^{2} n_{1}\right) .
$$


Clearly $P(\mathbf{n}) \geq P(\mathbf{n}, \varepsilon)$ where $P(\mathbf{n}, \varepsilon)$ is the contribution to the integral in the formula for $P(\mathbf{n})$, (see Lemma 1.2), coming from $D$, i.e.

$$
P(\mathbf{n}, \varepsilon)=\overbrace{\int \ldots \int}^{n_{1}}\left(\prod_{\mathbf{x} \in D}^{n_{1}} \int_{0}^{1} \prod_{i \neq j}\left(1-x_{i} y_{j}\right) d y_{j}\right) \prod_{h=1}^{n_{1}} \bar{x}_{h}^{n_{2}-n_{1}} d \mathbf{x} .
$$

Here, using (3.36), and then (3.33), (3.35),

$$
\begin{aligned}
\prod_{h=1}^{n_{1}} \bar{x}_{h}^{n_{2}-n_{1}} & =\exp \left(-\left(n_{2}-n_{1}\right) \sum_{h}\left(x_{h}+\frac{x_{h}^{2}}{2}+O\left(x_{h}^{3}\right)\right)\right) \\
& \geq \exp \left(-\left(n_{2}-n_{1}\right) s+\frac{\left(n_{2}-n_{1}\right) t}{2}\left(1+O\left(\sigma_{n_{1}}\right)\right)\right) \\
& =\exp \left(-\left(n_{2}-n_{1}\right) s+\frac{\left(n_{2}-n_{1}\right) s^{2}}{n_{1}}+O(\varepsilon)\right)
\end{aligned}
$$

as the last fraction is uniformly bounded for $s$ meeting (3.33). Likewise

$$
\begin{aligned}
\prod_{i \neq j}\left(1-x_{i} y_{j}\right) & \geq \prod_{i=1}^{n_{1}} \exp \left(-y_{j} s_{j}-y_{j}^{2} t \frac{1+O\left(\sigma_{n_{1}}\right)}{2}\right) \\
& =\prod_{i=1}^{n_{1}} \exp \left(-y_{j} s_{j}-y_{j}^{2} \frac{t}{2}+O\left(\sigma_{n_{1}}^{2}\right)\right)
\end{aligned}
$$

where

$$
s_{j}:=\sum_{i \neq j} x_{i}=s-x_{j}=s\left(1-O\left(n_{1}^{-1} \log n_{1}\right)\right) .
$$

Since

$$
s_{j}=s \exp \left(\log \left(1-\frac{x_{j}}{s}\right)\right)=s \exp \left(-\frac{x_{j}}{s}+O\left(x_{j}^{2} / s^{2}\right)\right),
$$

we have

$$
\prod_{j} s_{j}=e^{-1} s^{n_{1}} \exp \left(O\left(t s^{-2}\right)\right)=e^{-1} s^{n_{1}}\left(1+O\left(n_{1}^{-1}\right)\right) .
$$

Therefore, for each $j$,

$$
\begin{aligned}
& I_{j}(\mathbf{x}):=\int_{0}^{1} \prod_{i \neq j}\left(1-x_{i} y_{j}\right) d y_{j} \geq\left(1+O\left(\sigma_{n_{1}}^{2}\right)\right) \int_{0}^{1} \exp \left(-y s_{j}-y^{2} t / 2\right) d y \\
& \geq\left(1+O\left(\sigma_{n_{1}}^{2}\right)\right) s_{j}^{-1} \int_{0}^{s_{j}} \exp \left(-z-z^{2} \frac{t}{2 s_{j}^{2}}\right) d z \\
& =\left(1+O\left(\sigma_{n_{1}}^{2}\right)\right) s_{j}^{-1} \int_{0}^{s_{j}} \exp \left(-z-z^{2} \frac{t}{2 s^{2}}\right) d z \\
& \geq\left(1+O\left(\sigma_{n_{1}}^{2}\right)\right) s_{j}^{-1}\left(\int_{0}^{s} \exp \left(-z-z^{2} \frac{t}{2 s^{2}}\right) d z-x_{j} e^{-s-t / 2}\left(1+O\left(\sigma_{n_{1}}\right)\right)\right) .
\end{aligned}
$$


Here, as $t s^{-2}=O\left(n_{1}^{-1}\right)$, analogously to (3.10) we obtain

$$
1-e^{-s-t / 2}=\left(1-e^{-s}\right) \exp \left(\frac{t}{s^{2}} \frac{s^{2}}{2\left(e^{s}-1\right)}+O\left(n_{1}^{-2}\right)\right)
$$

also

$$
\frac{x_{j} e^{-s-t / 2}}{1-e^{-s-t / 2}}=\frac{x_{j}}{e^{s+t / 2}-1} \leq \frac{x_{j}}{e^{s}-1}=O\left(x_{j} / s\right)=O\left(n_{1}^{-1} \log n_{1}\right) .
$$

So it follows easily that

$$
I_{j}(\mathbf{x}) \geq\left(1+O\left(\sigma_{n_{1}}^{2}\right)\right)\left[1-\frac{t / s^{2}}{1-e^{-s}} \int_{0}^{s} z e^{-z} d z-\frac{x_{j}}{e^{s}-1}+\frac{t}{s^{2}} \frac{s^{2}}{2\left(e^{s}-1\right)}\right] .
$$

Thus, using (3.38),

$$
\prod_{j} I_{j}(\mathbf{x}) \gtrsim e\left(\frac{1-e^{-s}}{s}\right)^{n_{1}} \exp \left(-\frac{n_{1} t / s^{2}}{1-e^{-s}} \int_{0}^{s} z e^{-z} d z-\frac{s}{e^{s}-1}+\frac{s^{2}}{2\left(e^{s}-1\right)} \frac{n_{1} t}{s^{2}}\right) \text {. }
$$

Recalling the constraint (3.35), we have: uniformly for $\mathrm{x} \in D$,

$$
\begin{aligned}
I(\mathbf{x}) & :=\prod_{j=1}^{n_{1}} \bar{x}_{j}^{n_{2}-n_{1}} I_{j}(\mathbf{x}) \gtrsim(1+O(\varepsilon)) e^{1-H(s)} I(s), \\
H(s) & :=\frac{n_{2}-n_{1}}{n_{1}} s^{2}-\frac{2}{1-e^{-s}} \int_{0}^{s} z e^{-z} d z+\frac{s^{2}-s}{e^{s}-1} \\
& =\frac{n_{2}-n_{1}}{n_{1}} s^{2}-2+\frac{s+s^{2}}{e^{s}-1} .
\end{aligned}
$$

And we already proved in the subsection 3.1 that, uniformly for $s$ satisfying (3.33), $H(s)=h(\mathbf{n})+o(1)$, with $h(\mathbf{n})$ given by (3.15).

To lower-bound $P(\mathbf{n}, \varepsilon)$ we integrate the RHS of (3.39), with $h(\mathbf{n})$ instead of $H(s)$, over $D$. To do so, we switch to $n_{1}$ new variables $u, v_{1}, \ldots, v_{n_{1}-1}$ :

$$
u=\sum_{j=1}^{n_{1}} x_{j}, \quad v_{j}=x_{j} s^{-1}, 1 \leq j \leq n_{1}-1 .
$$

Define also $v_{n_{1}}=x_{n_{1}} s^{-1}$. Clearly $0 \leq v_{j} \leq 1$ and $\sum_{j=1} v_{j}^{n_{1}}=1$. The Jacobian of $\left(x_{1}, \ldots, x_{n_{1}}\right)$ with respect to $\left(u, v_{1}, \ldots, v_{n_{1}-1}\right)$ is $u^{n_{1}-1}$. The constraints (3.33)-(3.35) become

$$
\begin{aligned}
& (1-\delta(\mathbf{n})) s(\mathbf{n}) \leq u \leq(1+\delta(\mathbf{n})) s(\mathbf{n}), \\
& v_{j} \leq(1+\varepsilon) \frac{\log n_{1}}{n_{1}}, \quad\left(1 \leq j \leq n_{1}\right) ; \quad \sum_{j=1}^{n_{1}} v_{j}=1, \\
& (1-\varepsilon) \frac{2}{n_{1}} \leq \sum_{j=1}^{n_{1}} v_{j}^{2} \leq(1+\varepsilon) \frac{2}{n_{1}} .
\end{aligned}
$$


BORIS PITTEL

Obviously, but crucially, none of these constraints involves both $u$ and $\mathbf{v}$. Therefore

$$
P(\mathbf{n}, \varepsilon) \geq \frac{(1+O(\varepsilon)) e^{1-h(\mathbf{n})}}{\left(n_{1}-1\right) !} \int_{s \in \mathcal{I}(\mathbf{n})} u^{-1} I(u) d u \cdot \int_{\mathbf{v} \in \mathcal{D}}\left(n_{1}-1\right) ! \prod_{j=1}^{n_{1}-1} d v_{j}
$$

where $\mathcal{D}$ denotes the set of all $\mathbf{v}=\left(v_{1}, \ldots, v_{n_{1}}\right)$ meeting the constraints (3.41) and (3.42). As we mentioned earlier, $\left(n_{1}-1\right)$ ! is the joint density of the subintervals lengths $L_{1}, \ldots, L_{n_{1}-1}$ in the random partition of the interval $[0,1]$ by $n_{1}-1$ points chosen uniformly at random. Therefore

$$
\begin{aligned}
& \int_{\mathcal{D}}\left(n_{1}-1\right) ! d \mathbf{v} \\
& \quad=\mathrm{P}\left(\left\{L_{n_{1}}^{+} \leq(1+\varepsilon) \frac{\log n_{1}}{n_{1}}\right\} \bigcap\left\{(1-\varepsilon) \frac{2}{n_{1}} \leq \sum_{j} L_{j}^{2} \leq(1+\varepsilon) \frac{2}{n_{1}}\right\}\right),
\end{aligned}
$$

which tends to 1 , as $n_{1} \rightarrow \infty$. Furthermore, it was proved in Section 3.1, (3.26), that

$$
\int_{s \in \mathcal{I}(\mathbf{n})} u^{-1} I(u) d u \sim \frac{1}{s(\mathbf{n})\left(n_{2}-n_{1}\right)\left(\begin{array}{l}
n_{2} \\
n_{1}
\end{array}\right)} .
$$

Thus, for every $\varepsilon>0$,

$$
P(\mathbf{n}) \geq P(\mathbf{n}, \varepsilon) \geq(1+O(\varepsilon)) \frac{\exp \left(-\frac{e^{s(\mathbf{n})}-1-s(\mathbf{n})}{e^{s(\mathbf{n}}-1}\right)}{\left(n_{1}-1\right) !\left(n_{2}-n_{1}\right)\left(\begin{array}{l}
n_{2} \\
n_{1}
\end{array}\right) s(\mathbf{n})},
$$

implying that

$$
P(\mathbf{n}) \gtrsim \frac{\exp \left(-\frac{e^{s(\mathbf{n})}-1-s(\mathbf{n})}{e^{s(\mathbf{n})}-1}\right)}{\left(n_{1}-1\right) !\left(n_{2}-n_{1}\right)\left(\begin{array}{l}
n_{2} \\
n_{1}
\end{array}\right) s(\mathbf{n})} .
$$

Combining this estimate with (3.32) we have

$$
P(\mathbf{n}) \sim \frac{\exp \left(-\frac{e^{s(\mathbf{n})}-1-s(\mathbf{n})}{e^{s(\mathbf{n})}-1}\right)}{\left(n_{1}-1\right) !\left(n_{2}-n_{1}\right)\left(\begin{array}{l}
n_{2} \\
n_{1}
\end{array}\right) s(\mathbf{n})} .
$$

Since $\mathrm{E}[S(\mathbf{n})]$, the expected value of $S(\mathbf{n})$, the number of stable matchings, is $P(\mathbf{n})\left(\begin{array}{l}n_{2} \\ n_{1}\end{array}\right) n_{1}$ !, we proved Theorem 1.3, if $n_{2}>n_{1} \rightarrow \infty$ then

$$
\mathrm{E}[S(\mathbf{n})] \sim \frac{n_{1} \exp \left(-\frac{e^{s(\mathbf{n})}-1-s(\mathbf{n})}{e^{s(\mathbf{n})}-1}\right)}{\left(n_{2}-n_{1}\right) s(\mathbf{n})}, \quad s(\mathbf{n})=\log \frac{n_{2}}{n_{2}-n_{1}} .
$$




\section{Proof of Theorem 1.4}

Recall that $\min _{\mathcal{M}} Q(\mathcal{M})$ is the wives' rank $Q\left(\mathcal{M}_{1}\right)$ in the men-optimal stable matching. So $Q\left(\mathcal{M}_{1}\right)$ is distributed as the total number of proposals by men to women. Now, analogously to the balanced case (Wilson [16]), $Q\left(\mathcal{M}_{1}\right)$ is stochastically dominated by $N$, the number of consecutive random throws of balls, a ball per throw, into $n_{2}$ boxes till the moment when there are exactly $n_{1}$ non-empty boxes. (The difference between $N$ and $Q\left(\mathcal{M}_{1}\right)$ is the total number of redundant proposals made by men to women who had rejected them earlier.) $N$ is distributed as the sum of $n_{1}$ independent Geometrics with success probabilities $p_{j}:=\frac{n_{2}-j}{n_{2}}, 0 \leq j \leq n_{1}-1$. So

$$
\mathrm{E}[N]=\sum_{j=0}^{n_{1}-1} \frac{1}{p_{j}}=n_{2}\left(H_{n_{2}}-H_{n_{2}-n_{1}}\right) \sim n_{2} s(\mathbf{n}),
$$

$s(\mathbf{n})=\log \frac{n_{2}}{n_{2}-n_{1}}$, if $n_{2}>n_{1} \rightarrow \infty$. It can be proved that $N$ is sharply concentrated around $\mathrm{E}[N]$. We will not do it, but instead will use Lemma 1.2 to prove that w.h.p. all $Q(\mathcal{M})$ are sharply concentrated around $n_{2} s(\mathbf{n})$.

Let us start with bounding $\min _{\mathcal{M}} Q(\mathcal{M})$ from below. To this end, observe first that, for $k \geq n_{1}$,

$$
\mathrm{P}\left(\min _{\mathcal{M}} Q(\mathcal{M}) \leq k\right) \leq\left(\begin{array}{l}
n_{2} \\
n_{1}
\end{array}\right) n_{1} ! \sum_{\kappa<k} P_{\kappa}(\mathbf{n}) ;
$$

$P_{\kappa}(\mathbf{n})$ is the probability that a generic injection from $\left[n_{1}\right]$ to $\left[n_{2}\right]$ is stable. We want to show the RHS is vanishing in the limit for $k=(1-\delta(\mathbf{n}))\left(n_{2}-1\right) s(\mathbf{n})$. Here $\delta(\mathbf{n})$ is defined in (3.27).

By Lemma 1.2.

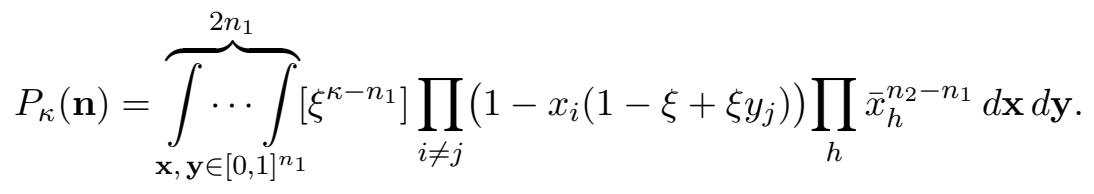

This identity is perfectly suited to application of Chernoff's method. Denoting $P_{k}^{-}(\mathbf{n}):=\sum_{\kappa \leq k} P_{\kappa}(\mathbf{n})$, we have

$$
\begin{aligned}
& P_{k}^{-}(\mathbf{n}) \leq \overbrace{\int_{\mathbf{x}, \mathbf{y} \in[0,1]^{n_{1}}} \cdots \int_{1 \leq i \neq j \leq n_{1}}^{2 n_{1}}} \inf \{\Phi(\xi, \mathbf{x}, \mathbf{y}): \xi \in(0,1]\} d \mathbf{x} d \mathbf{y}, \\
& \Phi(\xi, \mathbf{x}, \mathbf{y}):=\xi^{n_{1}-k} \prod_{\left.1-x_{i}\left(1-\xi+\xi y_{j}\right)\right) \prod_{h} \bar{x}_{h}^{n_{2}-n_{1}} d \mathbf{x} d \mathbf{y} .}(1-
\end{aligned}
$$


We will not try to determine the best $\xi=\xi(\mathbf{x}, \mathbf{y})$, and focus instead on a judicious choice of $\xi$ dependent only on $s=\sum_{i} x_{i}$. Using

$$
1-x_{i}\left(1-\xi+\xi y_{j}\right) \leq \exp \left(-x_{i}\left(1-\xi+\xi y_{j}\right)\right), \quad \bar{x}_{h} \leq e^{-x_{h}},
$$

and integrating over $\mathbf{y} \in[0,1]^{n_{1}}$, we obtain

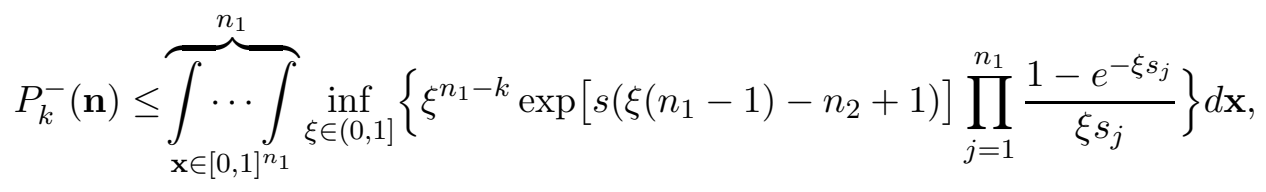

$\left(s_{j}=\sum_{i \neq j} x_{i}\right)$. Here, by (3.4) and $\xi \leq 1$,

$$
\prod_{j=1}^{n_{1}} \frac{1-e^{-\xi s_{j}}}{\xi s_{j}} \leq e^{2}\left(\frac{1-e^{-\xi s}}{\xi s}\right)^{n_{1}}
$$

so with the product replaced by its bound, the integrand becomes a function of $s$ only. Applying (3.2) in Lemma 3.1, we obtain then

$$
\begin{aligned}
P_{k}^{-}(\mathbf{n})= & O(1) \frac{1}{\left(n_{1}-1\right) !} \int_{0}^{n_{1}} \inf _{\xi \in(0,1]} \exp (H(s, \xi)) d s, \\
H(s, \xi):= & s\left[\xi\left(n_{1}-1\right)-n_{2}+1\right]+n_{1} \log \left(1-e^{-\xi s}\right) \\
& -\log s-k \log \xi .
\end{aligned}
$$

We are willing to bound $\inf _{\xi<1} e^{H(s, \xi)}$ by the value of $e^{H(s, \xi)}$ at a stationary point of $H(s, \xi)$ considered as a function of $\xi \in[0,1)$, hoping that this will the minimum point of this function. Now

$$
H_{\xi}(s, \xi)=s\left(n_{1}-1\right)+s n_{1}\left(e^{\xi s}-1\right)^{-1}-k \xi^{-1}=0,
$$

if $t:=\xi s$ satisfies an equation

$$
h(t)=k, \quad h(u):=u\left[\left(n_{1}-1\right)+n_{1}\left(e^{u}-1\right)^{-1}\right] .
$$

Since $h(0+)=n_{1}<k$ and $h(\infty)=\infty$, a root $t$ does exist, and it is unique, since $h^{\prime}(u) \geq h^{\prime}(0+)=\frac{n_{1}}{2}-1>0$. So ideally we would like to select $\xi(s)=t / s$ for $s \geq t$ and use the fall-back $\xi(s) \equiv 1$ for $s<t$. The technical issue here is necessity to deal with an implicitly defined $t$ as the root of $h(u)=k$. Observe that

$$
h(s(\mathbf{n}))=s(\mathbf{n})\left[\left(n_{1}-1\right)+\frac{n_{1}}{e^{s(\mathbf{n})}-1}\right]=\left(n_{2}-1\right) s(\mathbf{n})=\frac{k}{1-\delta(\mathbf{n})} .
$$

So let us try our luck with the explicit $t:=(1-\delta(\mathbf{n})) s(\mathbf{n})$, as an approximation for that implicit root, selecting $\xi(s)=t / s$ for $s \geq t$ and $\xi(s)=1$ for $s<t$. 
With $t=(1-\delta(\mathbf{n})) s(\mathbf{n})$, we have

$$
\begin{aligned}
& \int_{0}^{t} \exp \left(H(s, \xi(s)) d s=\int_{0}^{t} \exp (H(s, 1) d s\right. \\
& \quad=\int_{0}^{t} s^{-1} e^{-\left(n_{2}-n_{1}\right) s}\left(1-e^{-s}\right)^{n_{1}} d s \\
& \quad \leq \int_{z_{2}}^{1} z^{n_{2}-n_{1}-1}(1-z)^{n_{1}-1} d z \quad\left(z_{2}=e^{-(1-\delta(\mathbf{n})) s(\mathbf{n})}\right) \\
& \quad \leq{ }_{b} \varepsilon^{*}(\mathbf{n}) \int_{0}^{1} z^{n_{2}-n_{1}-1}(1-z)^{n_{1}-1} d z=\varepsilon^{*}(\mathbf{n}) \cdot \frac{n_{2} / n_{1}}{\left(n_{2}-n_{1}\right)\left(\begin{array}{c}
n_{2} \\
n_{1}
\end{array}\right)} ;
\end{aligned}
$$

Here

$$
\varepsilon^{*}(\mathbf{n})= \begin{cases}\varepsilon_{2}(\mathbf{n})=\exp \left(-0.99\left(n_{2}-n_{1}\right) e^{\delta(n) s(\mathbf{n})}\right), & \text { if } s(\mathbf{n}) \rightarrow \infty \\ \varepsilon(\mathbf{n})=\exp \left(-\theta\left(n_{1}^{1-2 a}\right)\right), & \text { if } s(\mathbf{n})=O(1)\end{cases}
$$

cf. (3.20) and (3.25).

Further, for $s \geq t$,

$$
\begin{aligned}
H(s, \xi(s))= & t\left(n_{1}-1\right)-s\left(n_{2}-1\right)+n_{1} \log \left(1-e^{-t}\right) \\
& -\log s-k \log \frac{t}{s} .
\end{aligned}
$$

So

$$
\begin{aligned}
\int_{t}^{\infty} \exp (H(s, \xi(s)) d s & \leq_{b} \exp \left(t\left(n_{1}-1\right)+n_{1} \log \left(1-e^{-t}\right)-k \log t\right) \\
& \times \int_{t}^{\infty} \exp \left(-s\left(n_{2}-1\right)\right) s^{k-1} d s
\end{aligned}
$$

Here, using $k=t\left(n_{2}-1\right)$,

$$
\begin{aligned}
& \int_{t}^{\infty} \exp \left(-s\left(n_{2}-1\right)\right) s^{k-1} d s \leq \int_{0}^{\infty} \exp \left(-s\left(n_{2}-1\right)\right) s^{k-1} d s \\
& =\frac{(k-1) !}{\left(n_{2}-1\right)^{k}} \leq{ }_{b}\left(\frac{k}{e\left(n_{2}-1\right)}\right)^{k-1} \leq \exp \left((k-1) \log t-t\left(n_{2}-1\right)\right),
\end{aligned}
$$

Therefore

$$
\begin{aligned}
& \int_{t}^{\infty} \exp \left(H(s, \xi(s)) d s \leq_{b} e^{\psi(t)},\right. \\
& \psi(s):=-s\left(n_{2}-n_{1}\right)+n_{1} \log \left(1-e^{-s}\right) .
\end{aligned}
$$


Here

$$
\begin{aligned}
\psi(s(\mathbf{n})) & =-n_{2} \log n_{2}+n_{1} \log n_{1}+\left(n_{2}-n_{1}\right) \log \left(n_{2}-n_{2}\right) \\
& =-\log \left(\frac{n_{2}^{n_{2}}}{n_{1}^{n_{1}}\left(n_{2}-n_{1}\right)^{n_{2}-n_{1}}}\right) \leq \log \left(\begin{array}{c}
n_{2} \\
n_{1}
\end{array}\right)^{-1}, \\
\psi^{\prime}(s(\mathbf{n})) & =-\left(n_{2}-n_{1}\right)+\left.\frac{n_{1}}{e^{s}-1}\right|_{s=s(\mathbf{n})}=0, \\
\psi^{\prime \prime}(s) & =-\frac{e^{s}}{\left(e^{s}-1\right)^{2}}
\end{aligned}
$$

in particular, for $s \in[t, s(\mathbf{n})]$,

$$
\psi^{\prime \prime}(s) \leq \psi^{\prime \prime}(s(\mathbf{n}))=-\frac{n_{2}\left(n_{2}-n_{1}\right)}{n_{1}} .
$$

Then

$$
\begin{aligned}
\psi(t) & \leq \psi(s(\mathbf{n}))+\frac{1}{2} \psi^{\prime \prime}(s(\mathbf{n}))(t-s(\mathbf{n}))^{2} \\
& =\log \left(\begin{array}{l}
n_{2} \\
n_{1}
\end{array}\right)^{-1}-\frac{n_{2}\left(n_{2}-n_{1}\right)}{2 n_{1}}(\delta(\mathbf{n}) s(\mathbf{n}))^{2}
\end{aligned}
$$

So

$$
\begin{aligned}
& \int_{t}^{n_{1}} \exp \left(H(s, \xi(s)) d s \leq \varepsilon^{* *}(\mathbf{n})\left(\begin{array}{c}
n_{2} \\
n_{1}
\end{array}\right)^{-1},\right. \\
& \varepsilon^{* *}(\mathbf{n})=\exp \left(-\frac{n_{2}\left(n_{2}-n_{1}\right)}{2 n_{1}}(\delta(\mathbf{n}) s(\mathbf{n}))^{2}\right) .
\end{aligned}
$$

Adding (4.7) and (4.11), we obtain

$$
\int_{0}^{n_{1}} \exp \left(H(s, \xi(s)) d s \leq_{b}\left[\varepsilon^{*}(\mathbf{n}) \frac{n_{2}}{n_{1}\left(n_{2}-n_{1}\right)}+\varepsilon^{* *}(\mathbf{n})\right]\left(\begin{array}{l}
n_{2} \\
n_{1}
\end{array}\right)^{-1} .\right.
$$

(1) If $s(\mathbf{n}) \rightarrow \infty$, then $n_{2} / n_{1} \rightarrow 1, \delta(\mathbf{n})=s(\mathbf{n})^{-b}, b<1$, and

$$
\begin{aligned}
\varepsilon^{*}(\mathbf{n}) & =\exp \left(-0.99\left(n_{2}-n_{1}\right) e^{s(\mathbf{n})^{1-b}}\right), \\
\varepsilon^{* *}(\mathbf{n}) & =\exp \left(-(0.5+o(1))\left(n_{2}-n_{1}\right) s(\mathbf{n})^{2(1-b)}\right),
\end{aligned}
$$

so that $\varepsilon^{*}(\mathbf{n}) \frac{n_{2}}{n_{1}\left(n_{2}-n_{1}\right)} \ll \varepsilon^{* *}(\mathbf{n})$. (2) If $s(\mathbf{n})=O(1)$, then $\delta(\mathbf{n})=n_{1}^{-a}$, $a<1 / 2$, and

$$
\varepsilon^{*}(\mathbf{n}) \frac{n_{2}}{n_{1}\left(n_{2}-n_{1}\right)}+\varepsilon^{* *}(\mathbf{n}) \leq \exp \left(-\theta\left(n_{1}^{1-2 a}\right)\right) .
$$


We conclude that the bound (4.4) implies

$$
\begin{aligned}
P_{k}^{-}(\mathbf{n}) & \leq_{b} \frac{\hat{\varepsilon}(\mathbf{n})}{\left(n_{1}-1\right) !}\left(\begin{array}{l}
n_{2} \\
n_{1}
\end{array}\right)^{-1}, \\
\hat{\varepsilon}(\mathbf{n}) & := \begin{cases}\exp \left(-0.49\left(n_{2}-n_{1}\right) s(\mathbf{n})^{2(1-b)}\right), & \text { if } s(\mathbf{n}) \rightarrow \infty, \\
\exp \left(-\theta\left(n_{1}^{1-2 a}\right)\right), & \text { if } s(\mathbf{n})=O(1) ;\end{cases}
\end{aligned}
$$

here $a \in(0,1 / 2), b \in(0,1)$. So, using (4.1), and

$$
\log n_{1} \leq s(\mathbf{n})+\log \left(n_{2}-n_{1}\right),
$$

we obtain

$$
\begin{aligned}
& \mathrm{P}\left(\min _{\mathcal{M}} Q(\mathcal{M}) \leq k\right) \leq P_{k}^{-}(\mathbf{n})\left(\begin{array}{l}
n_{2} \\
n_{1}
\end{array}\right) n_{1} !=O\left(n_{1} \hat{\varepsilon}(\mathbf{n})\right) \\
& \quad \leq_{b} \begin{cases}\exp \left(-0.48\left(n_{2}-n_{1}\right) s(\mathbf{n})^{2(1-b)}\right), & \text { if } s(\mathbf{n}) \rightarrow \infty, \\
\exp \left(-\theta\left(n_{1}^{1-2 a}\right)\right), & \text { if } s(\mathbf{n})=O(1),\end{cases}
\end{aligned}
$$

provided that $2(1-b)>1$, i.e. $b<1 / 2$.

We have proved

Lemma 4.1. Let $b<1 / 2$ in the definition (3.27) of $\delta(\mathbf{n})$. Then

$$
\mathrm{P}\left(\min _{\mathcal{M}} Q(\mathcal{M}) \leq(1-\delta(\mathbf{n})) n_{2} s(\mathbf{n})\right) \leq \begin{cases}\exp \left(-\theta\left(s(\mathbf{n})^{2(1-b)}\right)\right), & \text { if } s(\mathbf{n}) \rightarrow \infty, \\ \exp \left(-\theta\left(n_{1}^{1-2 a}\right)\right), & \text { if } s(\mathbf{n})=O(1) .\end{cases}
$$

We are about to prove that, on the other hand, w.h.p. $\max _{\mathcal{M}} Q(\mathcal{M}) \leq$ $k:=(1+\delta(\mathbf{n}))\left(n_{2}-1\right) s(\mathbf{n})$.

The argument runs parallel to the above proof of Lemma 4.1. Analogously to (4.1) and (4.4)-(4.5), we have

$$
\mathrm{P}\left(\max _{\mathcal{M}} Q(\mathcal{M}) \geq k\right) \leq\left(\begin{array}{c}
n_{2} \\
n_{1}
\end{array}\right) n_{1} ! P_{k}^{+}(\mathbf{n}), \quad P_{k}^{+}(\mathbf{n}):=\sum_{\kappa \geq k} P_{\kappa}(\mathbf{n}),
$$

and

$$
P_{k}^{+}(\mathbf{n}) \leq_{b} \frac{1}{\left(n_{1}-1\right) !} \int_{0}^{n_{1}} \inf _{\xi \in[1, \infty)} \exp (H(s, \xi)) d s,
$$

with $H(s, \xi)$ defined in (4.5). So now the Chernoff parameter $\xi$ exceeds 1 . Introduce $t=(1+\delta(\mathbf{n})) s(\mathbf{n})$, and set $\xi(s)=t / s$ for $s \leq t$ and $\xi(s)=1$ for $s>t$. 
Let us bound $\int_{0}^{n_{1}} \exp (H(s, \xi(s)) d s$. First, using (4.5), we have

$$
\begin{aligned}
& \int_{t}^{n_{1}} \exp \left(H(s, \xi(s)) d s=\int_{t}^{n_{1}} \exp (H(s, 1)) d s\right. \\
& \leq \int_{t}^{n_{1}} e^{-s\left(n_{2}-n_{1}\right)}\left(1-e^{-s}\right)^{n_{1}-1} d s .
\end{aligned}
$$

The same steps as in (3.17)-(3.24) deliver

$$
\begin{array}{r}
\int_{t}^{n_{1}} \exp \left(H(s, \xi(s)) d s \leq \varepsilon(\mathbf{n}) \frac{n_{2}}{n_{1}\left(n_{2}-n_{1}\right)}\left(\begin{array}{l}
n_{2} \\
n_{1}
\end{array}\right)^{-1},\right. \\
\varepsilon(\mathbf{n})= \begin{cases}\exp \left(-0.99\left(n_{2}-n_{1}\right) s(\mathbf{n})^{1-b}\right), & \text { if } s(\mathbf{n}) \rightarrow \infty, \\
\exp \left(-\theta\left(n_{1}^{1-2 a}\right)\right), & \text { if } s(\mathbf{n})=O(1) .\end{cases}
\end{array}
$$

For $s(\mathbf{n})=O(1)$ the factor $\varepsilon(\mathbf{n})$ is well suited for our needs. However, in the case $s(\mathbf{n}) \rightarrow \infty$ the power of $s(\mathbf{n})$ is too low. Fortunately, modifying and extending the argument for Lemma 4.1, we can double the power of $s(\mathbf{n})$.

Let us write

$$
\begin{aligned}
& e^{-s\left(n_{2}-n_{1}\right)}\left(1-e^{-s}\right)^{n_{1}-1}=e^{\psi_{1}(s)} \\
& \psi_{1}(s):=-s\left(n_{2}-n_{1}\right)+\left(n_{1}-1\right) \log \left(1-e^{-s}\right) .
\end{aligned}
$$

We have

$$
\begin{aligned}
& \psi_{1}^{(1)}(s)=-\left(n_{2}-n_{1}\right)+\left(n_{1}-1\right) \frac{1}{e^{s}-1}, \\
& \psi_{1}^{(2)}(s)=-\left(n_{1}-1\right) \frac{e^{s}}{\left(e^{s}-1\right)^{2}}, \\
& \psi_{1}^{(3)}(s)=\frac{2}{\left(e^{s}-1\right)^{3}}+\frac{3}{\left(e^{s}-1\right)^{2}} \leq \frac{3 e^{s}}{\left(e^{s}-1\right)^{3}},
\end{aligned}
$$

and $\psi_{1}^{(4)}(s)<0$. So $\psi_{1}(s)$ is concave, and attains its maximum at $s_{1}(\mathbf{n})=$ $\log \frac{n_{2}-1}{n_{2}-n_{1}}$. Clearly $\psi_{1}^{(1)}\left(s_{1}(\mathbf{n})\right)=0, s_{1}(\mathbf{n})<s(\mathbf{n})<t$, and

$$
\begin{aligned}
& \psi_{1}\left(s_{1}(\mathbf{n})\right)=-\log \frac{\left(n_{2}-1\right)^{n_{2}-1}}{\left(n_{1}-1\right)^{n_{1}-1}\left(n_{2}-n_{1}\right)^{n_{2}-n_{1}}} \leq-\log \left(\begin{array}{l}
n_{2}-1 \\
n_{1}-1
\end{array}\right), \\
& \psi_{1}^{(2)}\left(s_{1}(\mathbf{n})\right) \sim-\frac{n_{2}\left(n_{2}-n_{1}\right)}{n_{1}}, \\
& \psi_{1}^{(3)}(s) \leq \psi^{(3)}\left(s_{1}(\mathbf{n})\right) \lesssim \frac{3\left(n_{2}-n_{1}\right)^{2}}{n_{1}^{2}} \sim 3 s^{-2}(\mathbf{n}) .
\end{aligned}
$$

Since $t-s_{1}(\mathbf{n}) \sim t-s(\mathbf{n})=\delta(\mathbf{n}) s(\mathbf{n})$, and $\frac{n_{2}\left(n_{2}-n_{1}\right)}{n_{1}} \sim\left(n_{2}-n_{1}\right)$, we have

$$
\begin{aligned}
\psi_{1}(t) & \leq \psi_{1}\left(s_{1}(\mathbf{n})\right)-\frac{n_{2}-n_{1}}{2}(\delta(\mathbf{n}) s(\mathbf{n}))^{2}(1+o(1))+O\left(\delta^{3}(\mathbf{n}) s(\mathbf{n})\right) \\
& =\psi_{1}\left(s_{1}(\mathbf{n})\right)-\frac{n_{2}-n_{1}}{2}(\delta(\mathbf{n}) s(\mathbf{n}))^{2}(1+o(1)),
\end{aligned}
$$


and likewise

$$
\psi_{1}^{\prime}(t)=-\left(n_{2}-n_{1}\right) \delta(\mathbf{n}) s(\mathbf{n})(1+o(1)) \rightarrow-\infty .
$$

By concavity of $\psi_{1}(s)$, we also have $\psi_{1}(s) \leq \psi_{1}(t)+\psi_{1}^{\prime}(t)(t-s)$. Therefore, as $\delta(\mathbf{n})=s(\mathbf{n})^{-b}$ and $\frac{n_{2}}{n_{2}-1}=e^{s(\mathbf{n})}$,

$$
\begin{aligned}
& \int_{t}^{n_{1}} \exp \left(H(s, \xi(s)) d s \leq e^{\psi_{1}(t)} \int_{t}^{\infty} \exp \left(\psi_{1}^{\prime}(t)(s-t)\right) d s=\frac{e^{\psi_{1}(t)}}{-\psi_{1}^{\prime}(t)}\right. \\
& \leq{ }_{b} \frac{n_{2}}{n_{1}\left(n_{2}-n_{1}\right)}\left(\begin{array}{c}
n_{2} \\
n_{1}
\end{array}\right)^{-1} \exp \left(-\frac{n_{2}-n_{1}}{2} s(\mathbf{n})^{2(1-b)}(1+o(1))\right) \\
& \leq{ }_{b}\left(\begin{array}{c}
n_{2} \\
n_{1}
\end{array}\right)^{-1} \exp \left(-0.49\left(n_{2}-n_{1}\right) s(\mathbf{n})^{2(1-b)}\right),
\end{aligned}
$$

if $b<1 / 2$. Thus the bound in the first equation from (4.17) continues to hold for the much smaller

$$
\varepsilon(\mathbf{n}):=\exp \left(-0.49\left(n_{2}-n_{1}\right) s^{2(1-b)}(\mathbf{n})\right) .
$$

Turn to $s \in[0, t]$. Let us continue with the case $s(\mathbf{n}) \rightarrow \infty$. Analogously to (4.10), we have

$$
\int_{0}^{t} \exp \left(H(s, \xi(s)) d s \leq_{b} e^{\psi(t)}\right.
$$

We know that $\psi(s)$ attains its maximum at $s(\mathbf{n})$, and $\psi(s(\mathbf{n})) \leq-\log \left(\begin{array}{l}n_{2} \\ n_{1}\end{array}\right)$. Since $t-s(\mathbf{n})=\delta(\mathbf{n}) s(\mathbf{n})$, just like (4.19) we have

$$
\psi(t) \leq \psi(s(\mathbf{n}))-\frac{n_{2}-n_{1}}{2}(\delta(\mathbf{n}) s(\mathbf{n}))^{2}(1+o(1)) .
$$

So

$$
\int_{0}^{t} \exp \left(H(s, \xi(s)) d s \leq_{b}\left(\begin{array}{c}
n_{2} \\
n_{1}
\end{array}\right)^{-1} \exp \left(-0.49\left(n_{2}-n_{1}\right) s(\mathbf{n})^{2(1-b)}\right) .\right.
$$

By (4.20) and (4.24),

$$
\int_{0}^{n_{1}} \exp \left(H(s, \xi(s)) d s \leq_{b}\left(\begin{array}{l}
n_{2} \\
n_{1}
\end{array}\right)^{-1} \exp \left(-0.49\left(n_{2}-n_{1}\right) s(\mathbf{n})^{2(1-b)}\right) .\right.
$$

For the case $s(\mathbf{n})=O(1)$ we have $\delta(\mathbf{n})=n_{1}^{-a}, a<1 / 2$. The bound (4.22) still holds. Furthermore, using

$$
\psi^{\prime \prime}(s(\mathbf{n}))=-\frac{n_{2}\left(n_{2}-n_{1}\right)}{n_{1}}=\theta\left(n_{2}^{2} n_{1}^{-1}\right), \quad s(\mathbf{n})=\log \frac{n_{2}}{n_{2}-n_{1}} \geq \frac{n_{1}}{n_{2}},
$$

we have

$$
\begin{aligned}
\psi(t) & \leq \psi(s(\mathbf{n}))-\frac{\psi^{\prime \prime}(s(\mathbf{n}))}{2}(\delta(\mathbf{n}) s(\mathbf{n}))^{2}(1+o(1)) \\
& \leq \psi(s(\mathbf{n}))-\theta\left(n_{1}^{1-2 a}\right) .
\end{aligned}
$$


So

$$
\int_{0}^{t} \exp \left(H(s, \xi(s)) d s \leq\left(\begin{array}{c}
n_{2} \\
n_{1}
\end{array}\right)^{-1} \exp \left(-\theta\left(n_{1}^{1-2 a}\right)\right)\right.
$$

Combining this bound with (4.17) $(s(\mathbf{n})=O(1)$ case), we obtain

$$
\int_{0}^{n_{1}} \exp \left(H(s, \xi(s)) d s \leq_{b}\left(\begin{array}{c}
n_{2} \\
n_{1}
\end{array}\right)^{-1} \exp \left(-\theta\left(n_{1}^{1-2 a}\right)\right)\right.
$$

With the bounds (4.25) and (4.26) at hand, we argue exactly like in (4.12), (4.13) and (4.14) and establish

Lemma 4.2. In notations of Lemma 4.1.

$$
\mathrm{P}\left(\max _{\mathcal{M}} Q(\mathcal{M}) \geq(1+\delta(\mathbf{n})) n_{2} s(\mathbf{n})\right) \leq \begin{cases}\exp \left(-\theta\left(s(\mathbf{n})^{2(1-b)}\right)\right), & \text { if } s(\mathbf{n}) \rightarrow \infty \\ \exp \left(-\theta\left(n_{1}^{1-2 a}\right)\right), & \text { if } s(\mathbf{n})=O(1)\end{cases}
$$

\section{Proof of Theorem 1.5}

We need to show that, for $n_{2} \leq n_{1}^{3 / 2-d},(d<1 / 2)$, w.h.p. for all stable $\mathcal{M}$ 's the husbands' rank $R(\mathcal{M})$ is asymptotic to

$$
k=k(\mathbf{n}):=n_{1}^{2} f(s(\mathbf{n})), \quad f(x):=\frac{e^{x}-1-x}{x\left(e^{x}-1\right)} .
$$

Similarly to (4.1), for $k \geq n_{1}$ we have

$$
\begin{gathered}
\mathrm{P}\left(\min _{\mathcal{M}} R(\mathcal{M}) \leq k\right) \leq\left(\begin{array}{l}
n_{2} \\
n_{1}
\end{array}\right) n_{1} ! \sum_{\ell \leq k} P_{\ell}(\mathbf{n}), \\
\mathrm{P}\left(\max _{\mathcal{M}} R(\mathcal{M}) \geq k\right) \leq\left(\begin{array}{l}
n_{2} \\
n_{1}
\end{array}\right) n_{1} ! \sum_{\ell \geq k} P_{\ell}(\mathbf{n}),
\end{gathered}
$$

where $P_{\ell}(\mathbf{n})$ is the probability that a generic injection from $\left[n_{1}\right]$ to $\left[n_{2}\right]$ is stable, and the husbands' rank is $\ell$. Denote the first sum and the second sum $\mathcal{P}^{-}(k)$ and $\mathcal{P}^{+}(k)$ respectively. Let us bound these probabilities for some $k=k^{-}(\mathbf{n})$ and $k=k^{+}(\mathbf{n})$ respectively, such that $k(\mathbf{n}) \in\left[k^{-}(\mathbf{n}), k^{+}(\mathbf{n})\right]$. 
By the formula for $P_{\ell}(\mathbf{n})$ in Lemma 1.2 , we bound the sums:

$$
\begin{aligned}
& \mathcal{P}^{-}(k) \leq \overbrace{\int_{\mathbf{x}, \mathbf{y} \in[0,1]^{n_{1}}}^{2 n_{1}} \inf }^{n_{1}}\{\Psi(\eta, \mathbf{x}, \mathbf{y}): \eta \leq 1\} d \mathbf{x} d \mathbf{y} \\
& \mathcal{P}^{+}(k) \leq \overbrace{\int_{\mathbf{x}, \mathbf{y} \in[0,1]^{n_{1}}}^{2 n_{1}} \inf }^{2 n_{1}}\{(\eta, \mathbf{x}, \mathbf{y}): \eta \geq 1\} d \mathbf{x} d \mathbf{y} \\
& \Psi(\eta, \mathbf{x}, \mathbf{y}):=\eta^{n_{1}-k} \prod_{1 \leq i \neq j \leq n_{1}}\left(\bar{x}_{i} \bar{y}_{j}+x_{i} \bar{y}_{j}+\bar{x}_{i} y_{j} \eta\right) \cdot \prod_{h=1}^{n_{1}} \bar{x}_{h}^{n_{2}-n_{1}} .
\end{aligned}
$$

(a) Our first step is to dispense with the peripheral parts of the cube $[0,1]^{2 n_{1}}$ whose contribution to the integrals in (5.4) and (5.5) can be safely ignored. Fix $\gamma \in\left(\frac{1}{2}, 1\right), \rho \in(0,1)$, and define

$$
\begin{aligned}
& C_{1}=\left\{(\mathbf{x}, \mathbf{y}) \in[0,1]^{2 n_{1}}: \sum_{i} x_{i} y_{i} \geq n_{1}^{\gamma}\right\}, \\
& C_{2}=\left\{(\mathbf{x}, \mathbf{y}) \in[0,1]^{2 n_{1}}: \sum_{i} x_{i} \geq n_{1}-n_{1}^{\rho}\right\}, \\
& C_{0}=[0,1]^{2 n_{1}} \backslash\left(C_{1} \cup C_{2}\right) .
\end{aligned}
$$

Denote by $I^{ \pm}\left(C_{\alpha}\right)$ the contributions of $C_{\alpha}$ to the value of the integrals in (5.4) and (5.5), $(\alpha=0,1,2)$. For $\alpha=1,2$, choosing $\xi=\eta=1$ we have

$$
I^{ \pm}\left(C_{\alpha}\right) \leq \overbrace{\int_{\mathbf{x}, \mathbf{y} \in C_{\alpha}}^{2 n_{1}}}^{n_{1 \leq i \neq j \leq n_{1}}}\left(1-x_{i} y_{j}\right) d \mathbf{x} d \mathbf{y} .
$$

Here

$$
\begin{aligned}
\prod_{h=1}^{n_{1}} \bar{x}_{h}^{n_{2}-n_{1}} & \leq \exp \left(-\left(n_{2}-n_{1}\right) \sum_{h} x_{h}\right), \\
\log \prod_{1 \leq i \neq j \leq n_{1}}\left(1-x_{i} y_{j}\right) & \leq-\sum_{1 \leq i \neq j \leq n_{1}} x_{i} y_{j} \leq n_{1}-\left(\sum_{i} x_{i}\right)\left(\sum_{j} y_{j}\right),
\end{aligned}
$$

and for $C_{1}, s(\mathbf{x}):=\sum_{i} x_{i} \geq n_{1}^{\gamma}$, while by the Cauchy-Schwartz inequality and $x_{i}, y_{j} \leq 1$

$$
\left(\sum_{i} x_{i}\right)\left(\sum_{j} y_{j}\right) \geq\left(\sum_{i} x_{i}^{1 / 2} y_{i}^{1 / 2}\right)^{2} \geq\left(\sum_{i} x_{i} y_{i}\right)^{2} \geq n_{1}^{2 \gamma} .
$$

So

$$
I^{ \pm}\left(C_{1}\right) \leq \exp \left(n_{1}-n_{1}^{2 \gamma}-n_{1}^{\gamma}\left(n_{2}-n_{1}\right)\right)
$$


implying that the total contribution of $C_{1}$ to $\left(\begin{array}{l}n_{2} \\ n_{1}\end{array}\right) n_{1} ! \mathcal{P}^{ \pm}(k)$ is $\exp \left(-0.5 n_{1}^{2 \gamma}\right)$, i.e. $o\left(e^{-c n_{1}}\right)$ for every $c>0$ as $n_{2}>n_{1} \rightarrow \infty$, since $2 \gamma>1$. Using the definition of $C_{2}$ and the top inequality in (5.7), and integrating innermost over $\mathbf{y}$, we have: with $s^{*}=n_{1}-n_{1}^{\rho}$,

$$
\begin{aligned}
I^{ \pm}\left(C_{2}\right) & \leq \exp \left(-0.5 n_{1}\left(n_{2}-n_{1}\right)\right) \overbrace{\int_{\mathbf{x} \in[0,1]^{n_{1}}: s(\mathbf{x}) \geq s^{*}}^{n_{1}}}^{n_{1}}\left(\int_{0}^{1} e^{-s(\mathbf{x})} d y\right)^{n_{1}} d \mathbf{x} \\
& =\exp \left(-0.5 n_{1}\left(n_{2}-n_{1}\right)\right) \int_{s^{*}}^{n_{1}}\left(\frac{1-e^{-s}}{s}\right)^{n_{1}} f_{n_{1}}(s) d s,
\end{aligned}
$$

where $f_{n_{1}}(s)$ is the density of $\sum_{i} X_{i}$, the sum of the $n_{1}$ independent, [0,1]uniform random variables. By Lemma 3.1 .

$$
f_{n_{1}}(s)=\frac{s^{n_{1}-1}}{\left(n_{1}-1\right) !} \mathrm{P}\left(L_{n_{1}}^{+} \leq s^{-1}\right)
$$

where $L_{n_{1}}^{+}$is the length of the longest subinterval in the partition of $[0,1]$ by $n_{1}-1$ independent, uniform points. It was proved in [14 that

$$
\mathrm{P}\left(L_{n_{1}}^{+} \leq \zeta\right) \leq\left(n_{1} \zeta-1\right)^{n_{1}-1}, \quad \forall \zeta>n_{1}^{-1} .
$$

Therefore

$$
\begin{aligned}
I^{ \pm}\left(C_{2}\right) & \leq \frac{\exp \left(-0.5 n_{1}\left(n_{2}-n_{1}\right)\right)}{\left(n_{1}-1\right) !}\left(\frac{n_{1}^{\rho}}{n_{1}-n_{1}^{\rho}}\right)^{n_{1}-1} \int_{s^{*}}^{n_{1}} s^{-1} d s \\
& =O\left(\frac{\exp \left(-0.5 n_{1}\left(n_{2}-n_{1}\right)-0.5(1-\rho) n_{1} \log n_{1}\right)}{\left(n_{1}-1\right) !}\right),
\end{aligned}
$$

implying that the total contribution of $C_{2}$ to $\left(\begin{array}{l}n_{2} \\ n_{1}\end{array}\right) n_{1} ! P^{ \pm}(k)$ is $o\left(e^{-c n_{1}}\right)$ for every $c>0$ as $n_{2}>n_{1} \rightarrow \infty$, since $\rho<1$.

(b) Turn to the contribution of $C_{0}=[0,1]^{2 n_{1}} \backslash\left(C_{1} \cup C_{2}\right)$. Let $(\mathbf{x}, \mathbf{y}) \in C_{0}$. Similarly to (5.7), we obtain

$$
\begin{aligned}
& \log \left[\prod_{i \neq j}\left(\bar{x}_{i} \bar{y}_{j}+x_{i} \bar{y}_{j}+\bar{x}_{i} y_{j} \eta\right) \cdot \prod_{h} \bar{x}_{h}^{n_{2}-n_{1}}\right] \\
& \leq \sum_{i \neq j} y_{j}\left((\eta-1)-x_{i} \eta\right)-\left(n_{2}-n_{1}\right) s \\
& =-s(\eta) \sum_{j} y_{j}-\left(n_{2}-n_{1}\right) s+\eta \sum_{i} x_{i} y_{i},
\end{aligned}
$$

where $s=\sum_{i} x_{i}$, and

$$
s(\eta)=s-\left(n_{1}-1-s\right)(\eta-1)=n_{1}-1-\eta\left(n_{1}-1-s\right) .
$$


By the definition of $C_{0}$, the last term in (5.8) is $O\left(\eta \min \left(n_{1}^{\gamma}, s\right)\right)$. So $\Psi(\cdot)$ in (5.6) is bounded via

$$
\Psi(\eta, \mathbf{x}, \mathbf{y}) \leq \eta^{n_{1}-k} \exp \left[-s(\eta) \sum_{j} y_{j}-\left(n_{2}-n_{1}\right) s+O\left(\eta \min \left(n_{1}^{\gamma}, s\right)\right)\right]
$$

To upper-bound $I^{ \pm}\left(C_{0}\right)$, we will choose $\eta=\eta^{ \pm}$dependent on $s$ only. Of course, the admissible $\eta^{ \pm}(s)$ need to satisfy the conditions $\eta^{+}(s) \geq 1$ and $\eta^{-}(s) \leq 1$, respectively. Whatever our choice will be, integrating innermost with respect to $y$ and using the bound $f_{n_{1}}(s) \leq \frac{s^{n_{1}-1}}{\left(n_{1}-1\right) !}$, we obtain

$$
\begin{aligned}
I^{ \pm}\left(C_{0}\right) & \leq \frac{J^{ \pm}\left(C_{0}\right)}{\left(n_{1}-1\right) !} \\
J^{ \pm}\left(C_{0}\right) & =\int_{0}^{s^{*}} \exp \left(\mathcal{H}_{k}\left(s, \eta^{ \pm}\right)+O\left(\eta^{ \pm} \min \left(n_{1}^{\gamma}, s\right)\right)\right) d s, \\
\mathcal{H}_{k}(s, \eta) & :=\left(n_{1}-k\right) \log \eta+n_{1} \log \frac{1-\exp (-s(\eta))}{s(\eta)} \\
& +\left(n_{1}-1\right) \log s-\left(n_{2}-n_{1}\right) s ;
\end{aligned}
$$

here $s^{*}=n_{1}-n_{1}^{\rho}$, and $s(\eta)$ was defined in (5.9) .

An ideal $\eta^{ \pm}(s)$ is an admissible $\eta\left(s, k^{ \pm}\right)$that maximizes $\mathcal{H}_{k}(s, \eta),(k=$ $k^{ \pm}(\mathbf{n})$ ). (We hasten to add that the parameters $k^{ \pm}(\mathbf{n})$, that sandwich $k(\mathbf{n})$, will be defined shortly.)

As in the proof of Theorem 1.4, we are content to choose $\eta^{ \pm}(s)$ asymptotically close to a stationary point $\eta(s, k)$ of $H_{k}(s, \eta)$, considered as a function of $\eta$, provided that this point is admissible, of course. Now a stationary point is a root of

$$
\begin{aligned}
\frac{\partial \mathcal{H}_{k}(s, \eta)}{\partial \eta} & =\frac{n_{1}-k}{\eta}+n_{1} f(s(\eta))\left(n_{1}-1-s\right)=0, \\
f(x) & :=\frac{e^{x}-1-x}{x\left(e^{x}-1\right)}
\end{aligned}
$$

or, since $\eta\left(n_{1}-1-s\right)=n_{1}-1-s(\eta)$,

$$
k=n_{1}+n_{1}\left(n_{1}-1-s(\eta)\right) f(s(\eta)),
$$

if $s(\eta)=o\left(n_{1}\right)$. (It was the RHS in this formula that prompted us to come up with $k(\mathbf{n})=n_{1}^{2} f(s(\mathbf{n}))$.) Since $f(x)$ decreases with $x$, we hope for $s(\eta)$ to be asymptotic to

$$
s^{ \pm}(\mathbf{n})=\frac{s(n)}{1 \pm \delta(\mathbf{n})},
$$

for $=k^{ \pm}(\mathbf{n})$. Let us wait a bit more before we settle on the exact formulas for $k^{ \pm}(\mathbf{n})$. 
As $s(\eta)=n_{1}-1-\eta\left(n_{1}-1-s\right)$, we are thus led-informally, needless to say-to

$$
\eta^{ \pm}(s)=\frac{n_{1}-1-s^{ \pm}}{n_{1}-1-s}
$$

for $s$ such that $\eta^{+}(s) \geq 1$, and $\eta^{-}(s) \leq 1$, respectively; otherwise, we use the fall-back choices $\eta^{+}(s)=1$ and $\eta^{-}(s)=1$, respectively. To summarize, we have defined

$\eta^{-}(s)=\left\{\begin{array}{ll}1, & s \in\left[s^{-}, s^{*}\right], \\ \frac{n_{1}-1-s^{-}}{n_{1}-1-s}, & s<s^{-} ;\end{array} \quad \eta^{+}(s)= \begin{cases}\frac{n_{1}-1-s^{+}}{n_{1}-1-s}, & s \in\left[s^{+}, s^{*}\right], \\ 1, & s<s^{+} .\end{cases}\right.$

The "+" case. Suppose that $s \in\left[s^{+}, s^{*}\right]$. Then $s\left(\eta^{+}(s)\right)=s^{+}$, [see (5.9), (5.11)], and therefore $\mathcal{H}_{k^{+}}\left(s, \eta^{+}(s)\right)=H^{+}(s)$,

$$
\begin{aligned}
H^{+}(s):= & \left(n_{1}-k^{+}\right) \log \frac{n_{1}-1-s^{+}}{n_{1}-1-s}+n_{1} \log \frac{1-e^{-s^{+}}}{s^{+}} \\
& +\left(n_{1}-1\right) \log s-\left(n_{2}-n_{1}\right) s .
\end{aligned}
$$

The function $H^{+}(s)$ is concave and its derivative

$$
\frac{d H^{+}(s)}{d s}=\frac{n_{1}-k^{+}}{n_{1}-1-s}+\frac{n_{1}-1}{s}-\left(n_{2}-n_{1}\right)
$$

vanishes at $s=s^{+}$if we define

$$
\begin{aligned}
& k^{+}(\mathbf{n})=n_{1}+\left(n_{1}-1-s^{+}(\mathbf{n})\right)\left(\frac{n_{1}-1}{s^{+}(\mathbf{n})}-\left(n_{2}-n_{1}\right)\right) \\
& =n_{1}+n_{1}\left(n_{1}-1-s^{+}(\mathbf{n})\right)\left(f(s(\mathbf{n}))+\frac{\left(1-n_{1}^{-1}\right) \delta(\mathbf{n})-n_{1}^{-1}}{s(\mathbf{n})}\right) .
\end{aligned}
$$

Now, by the definition of $\delta(\mathbf{n})$,

$$
\delta^{*}(\mathbf{n}):=\frac{\delta(\mathbf{n})}{s(\mathbf{n}) f(s(\mathbf{n}))}= \begin{cases}O\left(s(\mathbf{n})^{-b}\right), & \text { if } s(\mathbf{n}) \rightarrow \infty \\ O\left(n_{2} n_{1}^{-1-a}\right), & \text { if } s(\mathbf{n})=O(1)\end{cases}
$$

where $b<1$ and $a<1 / 2$. Since $n_{2} \leq n_{1}^{3 / 2-\sigma}$, this $n_{2} n_{1}^{-1-a}$ tends to zero, provided that, in addition, $a>1 / 2-\sigma$, which we assume. So from (5.13) we have

$$
k^{+}(\mathbf{n}) \leq\left(1+1.01 \delta^{*}(\mathbf{n})\right) n_{1}^{2} f(s(\mathbf{n})) .
$$

Since $d^{2} H^{+} / d s^{2}<-n_{1} / s^{2}$, we have then: for $s \geq s^{+}$,

$$
\begin{aligned}
H^{+}(s) & \leq \psi_{1}\left(s^{+}\right)-\frac{n_{1}}{2}\left(\frac{s-s^{+}}{s}\right)^{2}, \\
\psi_{1}(s) & =\left(n_{1}-1\right) \log \left(1-e^{-s}\right)-\left(n_{2}-n_{1}\right) s .
\end{aligned}
$$


There is still the remainder term $O\left(\eta^{+} \min \left(n_{1}^{\gamma}, s\right)\right)$ in (5.11). It is easy to check that, for $s \leq s^{*}=n_{1}-n_{1}^{\rho}$, we have

$$
-\frac{n_{1}}{2}\left(\frac{s-s^{+}}{s}\right)^{2}+O\left(\eta^{+} \min \left(n_{1}^{\gamma}, s\right)\right) \leq A s(\mathbf{n})
$$

for some constant $A=A(\rho, \gamma)$, provided that $\rho>\gamma$. And this condition can be met, because up to now there were only two conditions on $\rho$ and $\gamma$ : $\rho \in(0,1)$ and $\gamma \in(1 / 2,1)$. Only a minor modification of the argument from (4.18) to (4.19) is needed to conclude that

$$
\begin{aligned}
& \int_{s^{+}}^{s^{*}} \exp \left(\mathcal{H}_{k^{+}}\left(s, \eta^{+}\right)+O\left(\eta^{+} \min \left(n_{1}^{\gamma}, s\right)\right)\right) d s \leq n_{1} e^{\psi_{1}\left(s^{+}\right)+A s(\mathbf{n})} \\
& \leq \begin{cases}\exp \left(-0.49\left(n_{2}-n_{1}\right) s(\mathbf{n})^{2(1-b)}\right)\left(\begin{array}{l}
n_{2} \\
n_{1}
\end{array}\right)^{-1}, & \text { if } s(\mathbf{n}) \rightarrow \infty, \\
\exp \left(-\theta\left(n_{1}^{1-2 a}\right)\right)\left(\begin{array}{l}
n_{2} \\
n_{1}
\end{array}\right)^{-1}, & \text { if } s(\mathbf{n})=O(1) .\end{cases}
\end{aligned}
$$

Continuing with the "+" case, suppose that $s \in\left(0, s^{+}\right)$. Then we have $\eta^{+}(s)=1$, so that $s\left(\eta^{+}(s)\right)=s$, and $\mathcal{H}_{k+}(s, 1)=\psi_{1}(s)$. On $\left(0, s^{+}\right]$the function $\psi_{1}(s)$ attains its maximum at $s^{+}$. So the contribution of this interval to $J^{+}\left(C_{0}\right)$ in (5.11) is at most the bottom bound in (5.15). Thus the total contribution of $C_{0}$ to $\left(\begin{array}{l}n_{2} \\ n_{1}\end{array}\right) n_{1} ! \mathcal{P}^{+}\left(k^{+}\right)$is, order-wise, below the same bound. And we recall that the contribution of the peripheral domain $C_{1} \cup C_{2}$ is $e^{-c^{*} n_{1}}$, at most. So, by (5.3), we obtain

$$
\begin{aligned}
& \mathrm{P}\left(\max _{\mathcal{M}} R(\mathcal{M}) \geq\left(1+1.01 \delta^{*}(\mathbf{n})\right) n_{1}^{2} f(s(\mathbf{n}))\right) \\
& \leq \begin{cases}\exp \left(-0.49\left(n_{2}-n_{1}\right) s(\mathbf{n})^{2(1-b)}\right), & \text { if } s(\mathbf{n}) \rightarrow \infty, \\
\exp \left(-\theta\left(n_{1}^{1-2 a}\right)\right), & \text { if } s(\mathbf{n})=O(1) .\end{cases}
\end{aligned}
$$

The "-" case. Suppose that $s \in\left[s^{-}, s^{*}\right]$. Then $\eta^{-}(s)=1, s\left(\eta^{-}(s)\right)=s$, and therefore the contribution of $\left[s^{-}, s^{*}\right]$ to $J^{-}\left(C_{0}\right)$ is, at most,

$$
\int_{s^{-}}^{s^{*}} \exp \left(\psi_{1}(s)+O\left(\min \left(n_{1}^{\gamma}, s\right)\right)\right) d s .
$$

On $\left[s^{-}, s^{*}\right], \psi_{1}(s)$ attains its maximum at $s^{-}=\frac{s(\mathbf{n})}{1-\delta(\mathbf{n})}$, and similarly to the "+" case, the integral is bounded by the bottom bound in (5.15). 
Suppose finally that $s \in\left(0, s^{-}\right)$; this is where we will choose $k^{-}(\mathbf{n})$. Then $s\left(\eta^{-}(s)\right)=s^{-}$, and therefore

$$
\begin{aligned}
\mathcal{H}_{k^{-}}\left(s, \eta^{-}(s)\right)= & H^{-}(s)+O(1), \\
H^{-}(s):= & \left(n_{1}-k^{-}\right) \log \frac{n_{1}-1-s^{-}}{n_{1}-1-s}+n_{1} \log \frac{1-e^{-s^{-}}}{s^{-}} \\
& +\left(n_{1}-1\right) \log s-\left(n_{2}-n_{1}\right) s .
\end{aligned}
$$

Here the derivative

$$
\frac{d H^{-}(s)}{d s}=\frac{n_{1}-k^{-}}{n_{1}-1-s}+\frac{n_{1}-1}{s}-\left(n_{2}-n_{1}\right)
$$

vanishes at $s^{-}$if we define

$$
\begin{aligned}
& k^{-}(\mathbf{n})=n_{1}+\left(n_{1}-1-s^{-}(\mathbf{n})\right)\left(\frac{n_{1}-1}{s^{-}(\mathbf{n})}-\left(n_{2}-n_{1}\right)\right) \\
& =n_{1}+n_{1}\left(n_{1}-1-s^{-}(\mathbf{n})\right)\left(f(s(\mathbf{n}))-\frac{\left(1-n_{1}^{-1}\right) \delta(\mathbf{n})+n_{1}^{-1}}{s(\mathbf{n})}\right) .
\end{aligned}
$$

So from (5.17) we have

$$
k^{-}(\mathbf{n})>\left(1-1.01 \delta^{*}(\mathbf{n})\right) n_{1}^{2} f(s(\mathbf{n})) .
$$

Since $H^{-}(s)$ is concave and its derivative at $s^{-}$is zero, it attains maximum at $s^{-}$, which equals

$$
n_{1} \log \frac{1-e^{-s^{-}}}{s^{-}}+\left(n_{1}-1\right) \log s^{-}-\left(n_{2}-n_{1}\right) s^{-} \leq \psi_{1}\left(s^{-}\right) .
$$

Therefore the contribution of $\left[0, s^{-}\right]$to $J^{-}\left(C_{0}\right)$ is below the bottom RHS in (5.15).

We conclude that

$$
\begin{aligned}
& \mathrm{P}\left(\min _{\mathcal{M}} R(\mathcal{M}) \leq\left(1-1.01 \delta^{*}(\mathbf{n})\right) n_{1}^{2} f(s(\mathbf{n}))\right) \\
& \leq \begin{cases}\exp \left(-0.49\left(n_{2}-n_{1}\right) s(\mathbf{n})^{2(1-b)}\right), & \text { if } s(\mathbf{n}) \rightarrow \infty, \\
\exp \left(-\theta\left(n_{1}^{1-2 a}\right)\right), & \text { if } s(\mathbf{n})=O(1) .\end{cases}
\end{aligned}
$$

Combining (5.18) and (5.16), we complete the proof of Theorem 1.5.

\section{Rotations EXPOSED IN RANDOM STABlE MATCHINGS}

For $n_{1}=n_{2}$, Irving and Leather [8] proved the following deep result. For every stable matching $\mathcal{M}$ different from the men-optimal stable matching $\mathcal{M}_{1}$ there exists a sequence of stable matchings $\left\{\mathcal{M}^{(j)}\right\}_{1 \leq j \leq t}$ with $\mathcal{M}^{(1)}=$ $\mathcal{M}_{1}$ and $\mathcal{M}^{(t)}=\mathcal{M}$ such that each $\mathcal{M}^{(j+1)}$ is obtained from $\mathcal{M}^{(j)}$ via a rotation step. It involves a cyclically ordered sequence of pairs $\left(m_{i}, w_{i}\right)$, $i \in[r]$, matched in $\mathcal{M}^{(j)}$, such that each woman $w_{i+1}$ is the best choice for the man $m_{i}$ among women to whom he prefers his wife $w_{i}$, and who prefer 
$m_{i}$ to their husbands in $\mathcal{M}_{j}$. Pairing each $m_{i}$ with $w_{i+1}$ we obtain the next stable matching $M^{(j+1)}$, in which each woman $w_{i}$ gets a better husband $m_{i-1}$, and all other women keep their husbands unchanged. As Rob Irving pointed out [6] this theorem holds for the case of $n_{1} \neq n_{2}$ as well. Thus, once we bound the expected total length of the rotations in all the stable matchings, we will obtain an upper bound for the expected number of all members with more than one stable partner.

Let $\left(\mathcal{M}, \mathcal{M}^{\prime}\right)$ be a given pair of matchings, with the same set of $n_{1}$ women, such that $\mathcal{M}^{\prime}$ is obtained from $\mathcal{M}$ by breaking up some pairs $\left(m_{1}, w_{1}\right), \ldots,\left(m_{r}, w_{r}\right)$ in $\mathcal{M}$ and pairing $m_{i}$ with $w_{i+1},\left(w_{r+1}:=w_{1}\right)$. Let $A$ denote the event that $\mathcal{M}$ is stable and $\left\{\left(m_{i}, w_{i}\right)\right\}$ is a rotation in $\mathcal{M}$, so that $\mathcal{M}^{\prime}$ is stable as well. By symmetry, $\mathrm{P}(A)$ depends only on $\mathbf{n}=\left(n_{1}, n_{2}\right)$, so we denote it $P(\mathbf{n}, r)$.

\section{Lemma 6.1.}

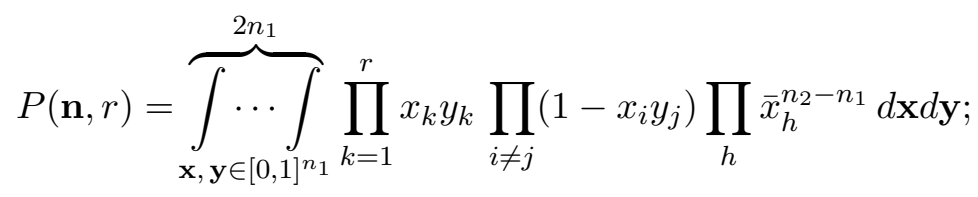

in the second product $j \neq i+1(\bmod r)$ for $1 \leq i \leq r$.

Proof. For $n_{1}=n_{2}$ this is Lemma 3.2 (a) in [14. Here $\mathcal{M}$ is the identity mapping from the men set $\left[n_{1}\right]$ to the women set $\left[n_{1}\right]$, and the rotation candidate is formed by the (man, woman) pairs $(1,1), \ldots,(r, r)$. The integrand is the probability that $\mathcal{M}$ is stable and that the sequence $(1,1), \ldots,(r, r)$ is indeed a rotation exposed in $\mathcal{M}$, conditioned on the event

$$
\begin{gathered}
X_{i, i+1}=x_{i},(1 \leq i \leq r),\left(X_{r, r+1}:=X_{r, 1}\right) ; \quad X_{i, i}=x_{i}, r+1 \leq i \leq n_{1}, \\
Y_{j, j}=y_{j},\left(1 \leq j \leq n_{1}\right)
\end{gathered}
$$

Corollary 6.2. Denoting $s=\sum_{i \in\left[n_{1}\right]} x_{i}$, and

$$
E_{1}(u):=\frac{1-e^{-u}}{u}, \quad E_{2}(u):=\frac{1-e^{-u}(1+u)}{u^{2}}
$$


we have

$$
\begin{aligned}
P(\mathbf{n}, r) & \leq \overbrace{b} \ldots \int_{\mathbf{x} \in[0,1]^{n_{1}}}^{n_{1}} E_{2}(s)^{r} E_{1}(s)^{n_{1}-r} e^{-\left(n_{2}-n_{1}\right) s}\left(\prod_{i=1}^{r} x_{i}\right) d \mathbf{x} \\
& =\frac{1}{\left(n_{1}+r-1\right) !} \int_{0}^{n_{1}} E_{2}(s)^{r} E_{1}(s)^{n_{1}-r} e^{-\left(n_{2}-n_{1}\right) s} s^{n_{1}+r-1} d s \\
& =\frac{1}{\left(n_{1}+r-1\right) !} \int_{0}^{n_{1}} s^{-1}\left(\frac{e^{s}-1-s}{e^{s}-1}\right)^{r}\left(1-e^{-s}\right)^{n_{1}} e^{-\left(n_{2}-n_{1}\right) s} d s .
\end{aligned}
$$

Proof. The proof mimics the derivation of the bound (5.8), and its subsequent transformation into a univariate integral, in [14].

The second factor in the integrand is below 1 . So from the proof of Theorem 1.3 in Section 3 the integral in (6.1) is asymptotically at most

$$
s(\mathbf{n})^{-1} \int_{0}^{n_{1}}\left(1-e^{-s}\right)^{n_{1}} e^{-\left(n_{2}-n_{1}\right) s} d s=\frac{1+o(1)}{s(\mathbf{n})\left(n_{2}-n_{1}\right)}\left(\begin{array}{c}
n_{2} \\
n_{1}
\end{array}\right)^{-1},
$$

$s(\mathbf{n})=\log \frac{n_{2}}{n_{2}-n_{1}}$. Now the total number of injections of $\left[n_{1}\right]$ into $\left[n_{2}\right]$ is $\left(\begin{array}{l}n_{2} \\ n_{1}\end{array}\right) n_{1}$ !, and the total number of cyclic sequences of $r$ matched pairs in an injection is $\left(\begin{array}{c}n_{1} \\ r\end{array}\right)(r-1) !=\frac{\left(n_{1}\right)_{r}}{r}$. Let $\mathcal{R}(\mathbf{n})$ be the total length of all the rotations exposed in all the stable matchings. By (6.1) and (6.2), we have then

$$
\begin{aligned}
& \mathrm{E}[\mathcal{R}(\mathbf{n})] \leq_{b} \frac{1}{s(\mathbf{n})\left(n_{2}-n_{1}\right)} \sum_{r \geq 2} \frac{n_{1} !\left(n_{1}\right)_{r}}{\left(n_{1}+r-1\right) !} \\
& =\frac{n_{1}}{s(\mathbf{n})\left(n_{2}-n_{1}\right)} \sum_{r \geq 2} \frac{\left(n_{1}\right)_{r}}{\left(n_{1}+r-1\right)_{r-1}} \\
& \leq \frac{n_{1}}{s(\mathbf{n})\left(n_{2}-n_{1}\right)} \sum_{r \geq 2} \exp \left(-\frac{r^{2}}{n_{1}}\right) \leq_{b} \frac{n_{1}^{3 / 2}}{s(\mathbf{n})\left(n_{2}-n_{1}\right)} .
\end{aligned}
$$

Now $n_{1}^{-1} R(\mathbf{n})$ is certainly an upper bound for both $m(\mathbf{n})$, the fraction of men, and $w(\mathbf{n})$, the fraction of women, with more than one stable partner. It is easy to check that, given $n_{1}$, the denominator $s(\mathbf{n})\left(n_{2}-n_{1}\right)$ is strictly increasing with $n_{2}$, and that

$$
n_{2}-n_{1}=\left[n_{1}^{1 / 2}\left(\log n_{1}\right)^{-\gamma}\right],(\gamma<1) \Longrightarrow \frac{n_{1}^{1 / 2}}{s(\mathbf{n})\left(n_{2}-n_{1}\right)} \rightarrow 0 .
$$

Thus we proved

Theorem 6.3. If $n_{2}-n_{1} \geq n_{1}^{1 / 2}\left(\log n_{1}\right)^{-\gamma},(\gamma<1)$, then $\lim m(\mathbf{n})=$ $\lim w(\mathbf{n})=0$, in probability. 
Aknowledgment. I owe debt of genuine gratitude to Jennifer Chayes for suggesting that the breakthrough results in [1] might warrant a follow-up research. I am grateful to Itai Ashlagi and Yash Kanoria for their encouraging interest in this work. I thank Rob Irving for his patient explanation of why the fruitful notion of rotations survives the transition from the classic case $n_{1}=n_{2}$ to the more general case $n_{1} \neq n_{2}$.

\section{REFERENCES}

[1] I. Ashlagi, Y. Kanoria and J. D. Leshno, Unbalanced random matching markets: the stark effect of competition, J Polit. Economy, forthcoming. Online Appendices A, B, C. Available at http://web.stanford.edu/ iashlagi/

[2] J. Chayes, Personal communication.

[3] W. Feller, An introduction to probability theory and its applications, 2nd edition, (1971).

[4] D. Gale and L. S. Shapley, College admissions and the stability of marriage, Amer Math Monthly 69 (1962) 9-15.

[5] D. Gusfield and R. W. Irving, The stable marriage problem, Structure and algorithms, Foundations of Computing Series (1989).

[6] R. W. Irving, Personal communication.

[7] G. Kalai, Combinatorics and more, https://gilkalai.wordpress.com/.

[8] R. W. Irving and P. Leather, The complexity of counting stable marriages, SIAM J Comput 15 (1986) 655-667.

[9] D. E. Knuth, Stable marriage and its relation to other combinatorial problems: an introduction to the mathematical analysis of algorithms, CRM Proceedings and Lecture notes (1996).

[10] D. E. Knuth, R. Motwani and B. Pittel, Stable husbands, Random Struct Algorithms 1 (1990) $1-14$.

[11] C. Lennon and B. Pittel, On the likely number of solutions for the stable matching problem, Combin Probab Comput 18 (2009) 371-421.

[12] D. G. McVitie and L. B. Wilson, The stable marriage problem, Comm ACM 14 (1971) 486-490.

[13] B. Pittel, The average number of stable matchings, SIAM J Disc Math 2 (1989) 530-549.

[14] B. Pittel, On likely solutions of a stable marriage problem, Ann Appl Probab 2 (1992) 358-401.

[15] B. Pittel, L. Shepp and E. Veklerov, On the number of fixed pairs in a random instance of the stable marriage problem, SIAM J. Discrete Math. 21 (2007) 947-958.

[16] L. B. Wilson, An analysis of the stable marriage assignment problem, BIT 12 (1972) 569-575.

Department of Mathematics, The Ohio State University, Columbus, Ohio 43210, USA

E-mail address: bgp@math.ohio-state.edu 